\title{
Heterogeneous Cloud Radio Access Networks: Enhanced Time Allocation for Interference Mitigation
}

\author{
Imad Al-Samman, ${ }^{1}$ Reham Almesaeed $(D),{ }^{2}$ Angela Doufexi ${ }^{D},{ }^{1}$ and Mark Beach ${ }^{1}$ \\ ${ }^{1}$ Department of Electrical and Electronic Engineering, Communications Systems and Networks Group, University of Bristol, \\ BS8 1UB, UK \\ ${ }^{2}$ Department of Computer Engineering, IT College, University of Bahrain, Bahrain
}

Correspondence should be addressed to Reham Almesaeed; ralmesaeed@uob.edu.bh

Received 4 February 2018; Revised 14 August 2018; Accepted 2 September 2018; Published 10 October 2018

Academic Editor: Jesus Fontecha

\begin{abstract}
Copyright (C) 2018 Imad Al-Samman et al. This is an open access article distributed under the Creative Commons Attribution License, which permits unrestricted use, distribution, and reproduction in any medium, provided the original work is properly cited.

Responding to the unprecedented challenges imposed by the $5 \mathrm{G}$ technologies, mobile operators have given significant attention to Heterogeneous Cloud Radio Access Networks (H-CRAN) due to their beneficial features of performing optimization, cost effectiveness, and improving spectral and energy efficiency performance. H-CRAN inherits the attractive benefits of Heterogeneous Networks (HetNet) and the cloud computing by facilitating interference mitigation, scalability, and radio resource control. Consequently, $\mathrm{H}$-CRAN is proposed in this article as a cost-effective potential solution to alleviate intertier interference and improve cooperative processing gains in HetNets by employing cloud computing. H-CRAN can provide efficient resource sharing at the spectrum, network, and infrastructure levels. Therefore, this article proposes H-CRAN cooperative interference mitigation method that enhances the time sharing among Radio Remote Heads (RRH) users. The study proposes an enhanced Almost Blank Subframe (ABSF) technique to increase the SINR and throughput of the small-cell (low power base station) and macrocell users. Simulation results show that the proposed Dynamic Programming-Diverse Almost Blank Subframe (ABSF) Pattern (DP-DAP) scheme improved the macro- and small-cell users up to $56 \%$ and $35 \%$, respectively, as compared to other state-of-the-art ABSF schemes.
\end{abstract}

\section{Introduction}

C-RAN is recognized as one of the key enabling technologies for future $5 \mathrm{G}$ communication systems because of its ability to fulfil the new requirements anticipated in $5 \mathrm{G}$ systems. C-RAN was first proposed in 2009 with further investigations and subsequent trials by joint operators and academic consortia $[1,2]$. It will facilitate and accommodate 5G technologies such as large scale antenna systems, full duplex, and ultra-dense networks due to its centralization nature and has the flexibility and scalability of a cloud-based implementation. In C-RAN systems, the large number of low cost RRHs are scattered over the network that provide basic wireless coverage, and connected through fronthaul links to a central processing unit called Base Band Unit (BBU) pool. The BBU pool is located in a centralized site and consists of time varying soft BBUs dynamically reconfigured. This architecture has several advantages. First of all, by locating the RRH closer to the user locations, a higher system capacity and lower power consumption can be achieved. In addition, the virtual base stations can work together in a large physical BBU pool and easily share the traffic data, signalling, and the channel state information of the User Equipment (UE), resulting in better spectral efficiency, since more efficient joint processing and scheduling techniques can be implemented. Secondly, the centralized BBU processing facilitates the cooperative processing techniques which mitigate interference issues as investigated in [3].

Most recently, the concept of $\mathrm{H}$-CRAN has been proposed as a cost-effective technology which is backward compatible with different kinds of C-RANs and HetNets. The motivation of H-CRANs is to embed cloud computing technology into HetNets to realize the large scale cooperative signal processing and networking functionalities [4]. 
Therefore, H-CRAN inherits the benefits of collective radio resources control and scalability, cooperative processing, and networking techniques. One key principle behind H-CRAN based $5 \mathrm{G}$ systems is that the control plane and the user plane are decoupled. The macrobase stations are used mainly to deliver control signalling and provide seamless coverage, while the RRHs are used to provide higher transmission rates in the hot spots. Therefore, $\mathrm{H}-\mathrm{CRAN}$ was conceived to enhance performance by incorporating cloud computing into HetNets to accomplish large scale signal processing through advance physical layer processing techniques such as massive Multiple-Input-Multiple Output (MIMO) techniques [4], where the cloudification enables the H-CRAN cooperative radio resource management and cooperative self-organizing network to schedule resources efficiently. Integrating two separate network architectures in H-CRAN imposes certain challenges which require dynamic resource sharing at three levels: spectrum, infrastructure, and network [5]. In the spectrum sharing part, the H-CRAN facilitates improved spectral efficiency through interference mitigation which is the focus of this article and dynamic spectrum access. At each of the aforementioned levels, the H-CRAN enables resource sharing benefits by providing dynamic pool of spectral resources, enhanced infrastructure coverage, and virtual networks tailored to particular service goals. Resource sharing in spectrum has been studied in $[6,7]$, while the infrastructure and network sharing are investigated in $[8,9]$.

The C-RAN has been deployed in HetNet environment to resolve intercell interference by facilitating spectrum and time-sharing techniques as discussed in Section 2.1. For example, spectrum sharing can be performed through allocation of channels and resource blocks in the Long-Term Evolution (LTE) frames. The available slots of the spectrum can also be shared dynamically, where the allocation unit can be expressed as certain power level, time slot, space, and code being shared and computed at the BBU. HetNet has also been considered as an effective method in achieving high spectral efficiency by Third Generation Partnership Project (3GPP) releases 10 and beyond. However, coordination across network tiers is necessary to mitigate interference caused by cells using the same spectrum. This can be achieved in the frequency domain by careful spectrum division and employing carrier aggregation, while in the time domain this is achieved by the ABSF technique. Enabling frequency and time-sharing techniques requires high connectivity mechanisms between the base stations (BSs) across the tiers. HCRAN will enable direct application of spectrum and timesharing techniques because the processing of the multitier cells and the orchestration are performed in the same BBUs pool. The flexibility of H-CRAN enables spectrum to be shared in a more efficient manner compared to alternative approaches. In LTE, sharing occurs in a resource block or in a subframe, whereas the joint processing supported by the C-RAN allows sharing at the symbol level. More efficient sharing enables better adaptation to different operators' needs resulting in improved resource utilization. This article focuses on proposing C-RAN cooperated time-domain interference mitigation technique between different cell tiers.
Despite the potential advantages of H-CRAN, they still require long-term development before they can be widely deployed. One of the main challenges that impacts the implementation of H-CRAN is the high demand on the data transmission through the backhaul links (optical or wireless links). The backhaul capacity is a challenge that limits the RRHs from making full use of the available radio resources.

The reminder of this article is outlined as follows. Motivation, related researches, and author's contribution are introduced in following section. Then the proposed H-CRAN cooperative interference mitigation model is discussed in Section 3, followed by the network and simulation models in Section 4. Detailed discussion on simulation results in provided inn Section 5. Finally, the conclusions are presented.

\section{Background Theory}

2.1. Relevant Work. In this article, we consider a H-CRAN model which allows centralized management of system radio resources to efficiently divide the spectrum between the deployed RRHs in the different cellular network tiers. The availability of UE and other system related information at the BBU will also be used to propose an enhanced time-sharing mechanism for users across the network based on the wellknown ABS methodology.

As discussed previously, the H-CRAN architecture with the cloud computing capabilities can be fully utilized to mitigate the inference and improve spectrum efficiency. In the literature, this area has been studied extensively, where a lot of state-of-the-art research studies on C-RAN and HetNet have been performed to investigate the H-CRAN capabilities. Examples of those include the work performed in [4], where the authors have proposed H-CRAN based solutions to dynamically control the amount of resources allocated to end users. This work trades off between fairness and throughput. In addition, in [10] a new cellular architecture of cloud cooperated HetNet is proposed to operate in multiband systems, where simulation results showed the effectiveness of the proposed architecture in achieving higher system rate than single band network. Recent work investigating resource sharing in C-RAN cooperated environments includes the work in [11], where the authors have proposed a novel multiresource sharing scheme based on the Indian buffet game, by constantly monitoring the C-RAN system conditions and adaptively responding in a distributed manner. Resource sharing in C-RAN cooperated environments, particularly spectrum sharing has also been investigated in [6]. The study reviewed the different types of spectrum bands for LTE/LTEAdvanced systems and focused on the licensed-shared access concept as a spectrally efficient solution for spectrum access. Moreover, the central processing aspects of C-RAN with a Heterogonous Networks (HetNet) architecture enable new methods to resolve intertier interference efficiently through resource sharing at the spectrum, infrastructure and networks levels as investigated in [12]. For each of the aforementioned levels, the study discussed the benefits, challenges, and highlighted the key enabling technologies that make resource sharing in C-RAN feasible. In addition, the authors 


\begin{tabular}{|l|l|l|l|l|l|l|l|l|l|l|l|l|l|l|l|l|l|l|l|l|l|l|l|l|l|l|l|l|l|}
\hline $\mathbf{0}$ & $\mathbf{1}$ & $\mathbf{2}$ & $\mathbf{3}$ & $\mathbf{4}$ & $\mathbf{5}$ & $\mathbf{6}$ & $\mathbf{7}$ & $\mathbf{8}$ & $\mathbf{9}$ & $\mathbf{0}$ & $\mathbf{1}$ & $\mathbf{2}$ & $\mathbf{3}$ & $\mathbf{4}$ & $\mathbf{5}$ & $\mathbf{6}$ & $\mathbf{7}$ & $\mathbf{8}$ & $\mathbf{9}$ & $\mathbf{0}$ & $\mathbf{1}$ & $\mathbf{2}$ & $\mathbf{3}$ & $\mathbf{4}$ & $\mathbf{5}$ & $\mathbf{6}$ & $\mathbf{7}$ & $\mathbf{8}$ & $\mathbf{9}$ \\
\hline
\end{tabular}

(a) Normal ABS: $1 \mathrm{ABS}$ out of 8 subframes, periodicity $=40 \mathrm{~ms}$

\begin{tabular}{|l|l|l|l|l|l|l|l|l|l|l|l|l|l|l|l|l|l|l|l|l|l|l|l|l|l|l|l|l|l|}
\hline $\mathbf{0}$ & $\mathbf{1}$ & $\mathbf{2}$ & $\mathbf{3}$ & $\mathbf{4}$ & $\mathbf{5}$ & $\mathbf{6}$ & $\mathbf{7}$ & $\mathbf{8}$ & $\mathbf{9}$ & $\mathbf{0}$ & $\mathbf{1}$ & $\mathbf{2}$ & $\mathbf{3}$ & $\mathbf{4}$ & $\mathbf{5}$ & $\mathbf{6}$ & $\mathbf{7}$ & $\mathbf{8}$ & $\mathbf{9}$ & $\mathbf{0}$ & $\mathbf{1}$ & $\mathbf{2}$ & $\mathbf{3}$ & $\mathbf{4}$ & $\mathbf{5}$ & $\mathbf{6}$ & $\mathbf{7}$ & $\mathbf{8}$ & $\mathbf{9}$ \\
\hline
\end{tabular}

(b) Normal ABS: 2 ABS out of 8 subframes, periodicity $=40 \mathrm{~ms}$

\begin{tabular}{|l|l|l|l|l|l|l|l|l|l|l|l|l|l|l|l|l|l|l|l|l|l|l|l|l|l|l|l|l|l|}
\hline $\mathbf{0}$ & $\mathbf{1}$ & $\mathbf{2}$ & $\mathbf{3}$ & $\mathbf{4}$ & $\mathbf{5}$ & $\mathbf{6}$ & $\mathbf{7}$ & $\mathbf{8}$ & $\mathbf{9}$ & $\mathbf{0}$ & $\mathbf{1}$ & $\mathbf{2}$ & $\mathbf{3}$ & $\mathbf{4}$ & $\mathbf{5}$ & $\mathbf{6}$ & $\mathbf{7}$ & $\mathbf{8}$ & $\mathbf{9}$ & $\mathbf{0}$ & $\mathbf{1}$ & $\mathbf{2}$ & $\mathbf{3}$ & $\mathbf{4}$ & $\mathbf{5}$ & $\mathbf{6}$ & $\mathbf{7}$ & $\mathbf{8}$ & $\mathbf{9}$ \\
\hline
\end{tabular}

(c) Normal ABS: 2 ABS out of 8 subframes, periodicity $=40 \mathrm{~ms}$

FIGURE 1: ABSF pattern.

in [13] have proposed a new cognitive radio sharing scheme by employing a coopetition game model that dynamically senses the spectrum based on the global game and efficiently reallocates the available spectrum. In a HetNet environment, where spectrum can be shared between different tiers, it is also necessary to deal with interference issues among tiers by reducing interference in the time domain, and this can be achieved by the ABS technique as investigated in $[14,15]$. In [14], the optimal amount of ABS for synchronous ABS configuration is studied by formulating the optimization as a network-wide utility maximization problem, while in [15], the authors proposed a calculation scheme for the optimal muting ratio based on parameters that can be estimated by the network.

Focusing on the different techniques that are proposed in the literature to mitigate intercell interference in HetNet environments, power control is one of the techniques that are used in 3GPP for handling a dominant interference scenario [16], while ABSF is another approach to manage intercell interference in the time domain [17]. Figure 1 shows an example of ABS patterns for normal ABSs. It illustrates normal ABS densities of $12.5 \%$ and $25 \%$, corresponding to $1 \mathrm{ABS}$ and 2 ABSs, respectively, per eight subframes. The main idea of this technique is that all BSs follow universal frequency reuse and are switched off for a fraction of frame to reduce interference experienced by neighbouring BSs. Our proposed interference mitigation scheme is an enhanced ABS scheme that allows more macrousers to selectively transmit during blank frames for higher throughput performance. The idea of spectrum sharing as a mechanism of addressing interference is not new; examples (include the fractional frequency reuse scheme) have been proposed in the literature $[18,19]$. In such schemes, the cell is divided into an inner region comprising users close to the BS and an outer region, which comprises users close to the cell edge. Neighbouring BSs can share frequencies used within inner region [20,21]. In terms of HetNet, there are two categories of interference control; the first is the multiband HetNet where, e.g., macro-BS operates at $2 \mathrm{GHz}$ band and pico-BSs at $3 \mathrm{GHz}$ or $60 \mathrm{GHz}$ band. In the second category, all BSs operate in the same frequency band which causes intercell interference. In this case, one of the proposed techniques in the literature to mitigate interference is to divide the spectrum into subbands to allocate them to macro- and lower-power BSs. The studies performed in this context include $[20,21]$, which are based on the idea of dividing the spectrum into shared and dedicated portions, for example, as in [20]. A frequency assignment scheme is proposed for femtocells considering practical issues such as hand-off, coverage area, and the spectrum division which is based on the calculated interference-limited coverage area of the cochannel femtocell.

2.2. Author's Contribution. In previous subsection, we presented recent studies in the area of intercell interference mitigation in both time and frequency domains. The authors of this article have proposed in [22] a C-RAN cooperated interference mitigation technique in the frequency domain; however in this article and for further enhancement of the former study, the authors are focusing on proposing a timedomain interference solution. Therefore, the goal of the study is to address the intercell interference in C-RAN based network, by proposing time-domain interference mitigation mechanism based on the well-known ABSF scheme. The proposed DP-DAP technique in this article works jointly with a previously proposed User Weighted Probability Algorithm (UWPA) frequency sharing technique to provide complete framework for intertier interference mitigation facilitated by C-RAN. The contribution and modifications proposed by the authors is summarized in the following points:

(1) Enhance the existing ABSF technique by proposing a novel macrocell sectorization mechanism to implement diverse-ABSF patterns.

(2) Propose a new algorithm for classifying victim and nonvictim users in current ABSF scheme.

(3) Utilize C-RAN functions to implement the proposed interference mitigation technique in HetNet scenario.

(4) Design joint DP-DAP and UWPA framework for intertier interference mitigation.

\section{Proposed C-RAN Cooperated Interference Mitigation Model in Time Domain}

The proposed ABSF-based scheme (referred to later as DP$\mathbf{D A P}$ ) is developed to mitigate the interference problem in HetNet networks facilitated by the C-RAN capabilities. The DP-DAP technique works jointly with the author's previously proposed UWPA technique [22] to further reduce the intercell interference. The UWPA is reviewed briefly in Section 3.1 to familiarize the reader with this model that provides solution to intercell interference in frequency 
domain. Then the DP-DAP algorithm is presented in detail in Section 3.2.

The main idea behind the DP-DAP technique is that the macrobase station (MBS) mutes a fraction of subframes to allow victim small-cell users to receive the signal with higher Signal-to-Noise Ratio (SINR) from small base station (SBS), while victim and nonvictim users are classified according to the proposed DP-DAP algorithm. For simplicity, this study only considers 1 MBS. The fraction of muted subframes out of the total number of subframes in a single frame is termed as a muting ratio which takes range of values within $\lceil 0,1\rceil$. The proposed DP-DAP scheme requires coordination between the three entities: MBS, SBSs, and macrousers, which is performed through C-RAN functions as explained in Section 3.2.

\subsection{Review of the Frequency Domain Interference Mitigation Model (UWPA)}

3.1.1. The UWPA: Key Principles. This subsection reviews a previously proposed spectrum sharing technique between macro- and small-cell users. The authors have previously proposed the UWPA spectrum sharing technique [22] which is joined with the work in this article to provide comprehensive solution for intertier interference mitigation. The UWPA exploits the centralized processing nature of the C-RAN to assign the optimum shared portion of the bandwidth across number of picocells which are classified as noninterfering cells with slight impact on macrousers, while the most severe interferers are allocated the dedicated chunk of the spectrum. The study results that are published in [22] show the proposed scheme to outperform the literature relevant studies. Sharing spectrum across different tiers is not a new area and has been addressed in [20,21] as discussed in [22]. However, both schemes rely on the SBS to select their spectrum usage mode based on the cell location and a threshold parameter, which divide the whole macroarea into inner and outer zones. The proposed UWPA is inspired by the work presented in [23], in which the authors have proposed a probability weighted scheme to divide the operating bandwidth efficiently between the MBS and SBSs. Their method proposed a classification mechanism for the small cells into shared or dedicated bandwidths, which is implemented in the allocation unit in the BBU pool. The SBSs that share the spectral resources with MBS are marked as interfering cells on the macrousers due to their severe impact. On the contrary, the dedicated cells are labelled noninterferers on the macrousers.

The proposed UWPA algorithm is based on two main stages; the first stage is an enhanced model for the algorithm proposed in [23]. However, this stage aims to increase the number of small-cell users using the shared spectrum, by considering small-cell density as an embedded factor in making the decision whether the cell is shared or dedicated. The algorithm assumes the presence of a class table stored in the data centre; this table includes user's class, the Quality of service (QoS), and SINR requirement for each macrouser and small-cell class. For simplicity, the macrousers are clustered in three class levels as illustrated in Table 1 and each level is associated with SINR threshold.
TABLE 1: SINR user requirements.

\begin{tabular}{lc}
\hline Class & Min macro user SINR \\
\hline Premium & $13 \mathrm{~dB}$ \\
Gold & $6 \mathrm{~dB}$ \\
Basic & $0 \mathrm{~dB}$ \\
\hline
\end{tabular}

The proposed UWPA scheme assumes configuration signalling that takes place every specific time frame according to the manufacturers and vendors. The Resource Intelligence Entity (RIE) in C-RAN centre (BBU pool) collects the channel conditions for small-cell users across all small cells. The small-cell user receives the pilot power from the MBS and other SBSs including the one serves the small-cell user to calculate the Reference Signal Received Power (RSRP) and Reference Signal Received Quality (RSRQ) as in LTE and reports it back to the C-RAN centre via the serving cell; this could occur by the added radio resource control signalling message. By collecting those measurements, the RIE is able to calculate the SINR for each small-cell user, then averaging their SINRs in a certain SBS to compute the mean SINR as LTE cell's key performance indicator, then compare it against the targeted SBS threshold. The SBSs are categorised onto three different classes in terms of Radio Frequency (RF) conditions. Cells' classes in this technique are considered as the following: the premium cell which should have the best coverage and relatively high mean SINR $(\sim 10$ $\mathrm{dB})$ as a threshold, the golden cell with less strict mean SINR requirements $(\sim 5 \mathrm{~dB})$, and the basic cells with the lowest requirements $(\sim 0 \mathrm{~dB})$, such that if the relevant SINR is greater than the class threshold, the SBS is listed in an initial list that created by the C-RAN of the SBSs that operate in the shared portion within the studied macrocell. Otherwise, the SBS's users cannot cope with high interference resulting in low SINR. Likewise, the macrousers receive pilot signal from the SBSs to calculate the relevant RSRP and RSRQ. They are reported afterwards to the C-RAN centre via MBS. The RIE in the centre can compute the intertier interference from each SBS on every macrouser. The RIE is assumed to have a table of the macrousers with their associated class, from which it can precisely set the maximum interference that each macrouser can experience in order to satisfy the class SINR requirement. For each macrouser, the RIE finds the list of SBSs that can use the shared spectrum.

In the second stage of the UWPA algorithm, the optimum set of SBSs is selected, which constitutes the list of SBSs that have the least intertier interference on macrousers. The selected list should have least interference impact on the premium macrousers, then golden, and finally the basic users.

UWPA is shown to outperform the considered literature state-of-the-art schemes since it accounts for both small cells densities and intertier interference. Exploiting the small-cell density results in a higher optimized shared ratio that results in turn in a higher portion of shared spectrum and more small cells to operate in shared bandwidth. However, when greater number of small cells operate in the shared part, higher intertier interference is experienced by macro- and small-cell users. Nevertheless, stage 2 of UWPA addresses this 
point as it looks up for the set of small cells with the least effect with respect to the interference power on macro- and small users.

3.1.2. The UWPA: Sample Results. The UWPA algorithm is compared with the following state-of the art schemes.

(A) A Hybrid Frequency Assignment for femtocells and coverage area analysis (HFAC) [20]: this scheme depends on the small-cell radius as a threshold to compare the power received from the MBS and each small-cell SBS in order to classify the small BSs according to the shared and dedicated spectrum portions.

(B) The Hybrid Spectrum Usage Algorithm (HSUA) [21]: this scheme relies on the geographic location of the SBS and its separation distance from the MBS. The vicinity of the MBS is divided into an inner and outer zone; small cells located in the inner part (close to MBS) operate in the dedicated spectrum, while the ones located in the outer zone (away from $M B S$ ) operate in the shared partition.

(C) Probability Weighted Based Algorithm (PWBA) [23]: the authors of this work considered only the impact of SBS on macrousers with one user per SBS and selected the final set of SBS to utilize shared spectrum as an intersection between SBS lists generated by the PWBA.

The throughput analysis of both small- and macrocell users is presented in [22] showing enhancement introduced by the UWPA technique. For example, Figure 2 illustrates the Cumulative Distribution Function (CDF) which is defined as the probability that a random variable $\mathrm{X}$ takes a value smaller than some specific value $\mathrm{xx}$. The CDFs are generated from Monte Carlo simulation, where the total number of 200 users are distributed in the macrocell vicinity, and users are either connected to the macro-BS or the pico-BS based on the higher received power criteria. The simulation is then repeated for five times in order to generate network realizations. It is observed from Figure 2 that for the smallcell user throughput, all schemes achieve close performance for the low throughput values, with UWPA scheme achieving slightly lower throughput compared to other schemes (e.g., less than $20 \mathrm{Mbps}$ ). However, for higher throughput levels, the proposed scheme UWPA gives better performance, for example, $74 \%$ of small-cell user's throughput is less than or equal $90 \mathrm{Mbps}$, where $88 \%$ achieved this rate for the case of HSUA scheme and $90 \%$ for the HFCA and PWBA schemes. In our proposed algorithm, the selection process of the final set of SBSs to operate in shared spectrum is implemented through several stages in order to maintain high QoS for premium, golden, and basic macrousers, respectively. Both HFAC and HSUA considered only the potential improvement of the SBS's spectral efficiency but do not take into account the intertier interference on macrousers, while $P W B A$ takes into consideration the interference at the macrousers. Nevertheless, the $P W B A$ algorithm works only for a limited number of macrousers as they assumed only 10

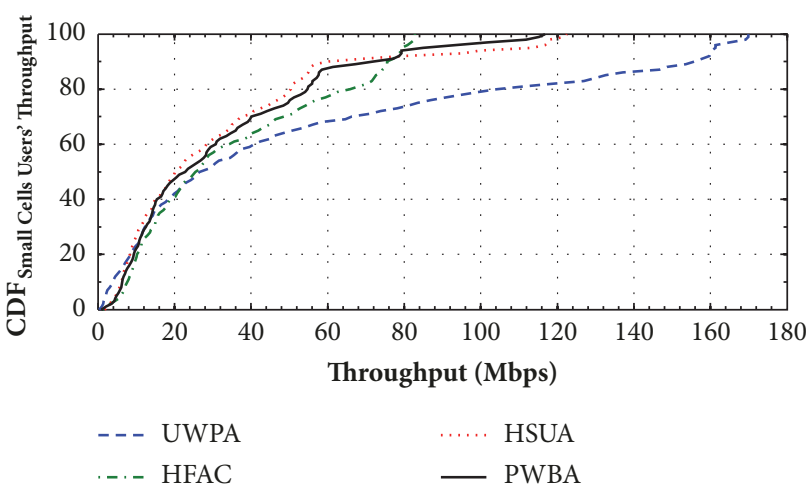

Figure 2: CDF of small-cell user's throughput.

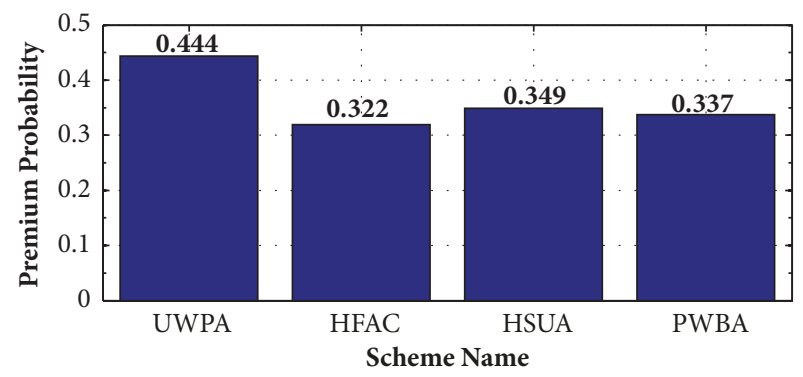

FIGURE 3: Macrousers QoS satisfaction ratios.

macrousers in their simulation [23], where the intersection stage of $P W B A$ minimises the number of SBSs to operate in the shared spectrum significantly in a reverse proportion with the number of macrousers. Thus, $P W B A$ is not feasible for a large number of macrousers.

On the other hand, given that QoS is considered in the $U W P A$, the performance is also measured in terms of the premium user's satisfaction ratio, which is the probability that a particular premium user achieves a data throughput equal to or greater than certain threshold as defined in

$$
P_{c}(T, U E p r e m i e r) \triangleq P(T h r>T),
$$

where $T$ is the throughput threshold based on the data rate requirements of the user, which is considered $20 \mathrm{Mbps}$. Figure 3 highlights the probability of premium users both macrousers and small users to achieve throughput higher than $20 \mathrm{Mbps}$ in all schemes. UWPA implementation places high priority for premium users which leads to higher service satisfaction in terms of PHY throughput. For example, the proposed UWPA achieved $45 \%$ satisfaction ratio compared to $32 \%, 35 \%$, and $34 \%$ for the HFAC, HSUA, and PWBA schemes, respectively.

\subsection{Proposed DP-DAP Technique}

3.2.1. Problem Formulation. Both MBS and SBS are assumed to follow a proportional fair scheduling approach. All smallcell users are assumed to have an equal opportunity being scheduled for the long run as Round Robin (RR) scheduler. Therefore, the average throughput for specific small-cell user 
(j) can be calculated as $\overline{r_{i j}}=r_{i j} / K_{i}$ where $K_{i}$ is the number of users in particular small base station $(j) S B S_{i}$. A user is classified as victim based on utility function $U$ defined in

$$
U=\prod_{i=1}^{m} \prod_{j=1}^{K_{i}} \bar{r}_{i j}=\frac{\prod_{i=1}^{m} \prod_{j=1}^{K_{i}} r_{i j}}{K_{i}}=\frac{\prod_{i=1}^{m} \prod_{j=1}^{K_{i}} r_{i j}}{\prod_{i=1}^{m} K_{i}^{K_{i}}}
$$

It can be deduced from (2) that when a new user joins a specific $S B S i$, the new $\mathrm{U}$ is defined as

$$
U_{\text {new }}(i, j)=U_{\text {old }} \cdot \frac{r_{i j} \cdot K_{i}^{K_{i}}}{\left(K_{i}+1\right)^{K_{i}+1}}
$$

The authors in [24] have proposed cell association mechanism based on the maximization utility function $U$ presuming $K_{i}$ is the number of small-cell users in $S B S_{i}$ that is viewed as $\left(K_{i}-1\right)$ original users plus the new added one. The authors have computed the maximal utility function by Dynamic Programming (DP) as in

$$
\begin{aligned}
& U_{\max }\left(K_{1}, K_{2}, \ldots, K_{m}\right) \\
& =\max \left\{\begin{array}{c}
U_{\max }\left(K_{1}, K_{2}, \ldots, K_{i-1}, \ldots, K_{m}\right) \cdot \frac{r_{i j} \cdot\left(K_{i}-1\right)^{K_{i}-1}}{\left(K_{i}\right)^{K_{i}}} \mid j \\
=\sum K_{i}
\end{array}\right\}
\end{aligned}
$$

The same strategy in calculating $U_{\max }$ by DP has inspired the authors in [14] to apply it in "victim UE Reselection" and it is considered the core mechanism in determining the victim users in this study. Presuming a particular $S B S_{i}$ that has certain number of users associated with it (regardless of what scheme used for association) and muting ratio $\alpha$. The aggregated cell data rate for a value of $\alpha$ (denoted as $\left.U_{i}^{t o t}(\alpha)\right)$ is calculated as the sum of two subutility functions $\left(U_{i}^{N}(\alpha), U_{i}^{V}(\alpha)\right)$ associated with nonvictim and victim users which are donated as $N_{i}$ and $V_{i}$ with density in $S B S_{i}$ as $N_{i}^{N}(\alpha)$ and $N_{i}^{V}(\alpha)$, respectively. $N_{i}$ users are scheduled specifically in $\boldsymbol{A B S}$ subframes, while $V_{i}$ users are in non- $\boldsymbol{A B S}$ subframes. Based on (2) and taking the log for its components, $U_{i}^{\text {tot }}(\alpha)$ can be rewritten as

$$
\begin{aligned}
U_{i}^{\text {tot }}(\alpha)= & U_{i}^{N}(\alpha)+U_{i}^{V}(\alpha) \\
= & \left\{\log _{2}(1-\alpha) \cdot N_{i}^{N}(\alpha)+\sum_{j=1}^{N_{i}^{N}(\alpha)} \log _{2}\left(R_{N j}^{i}\right)\right\} \\
& +\left\{\log _{2}(\alpha) \cdot N_{i}^{V}(\alpha)+\sum_{j=1}^{N_{i}^{V}(\alpha)} \log _{2}\left(R_{V j}^{i}\right)\right\}
\end{aligned}
$$

$R_{N j}^{i}$ and $R_{V j}^{i}$ in (5) are the average data rates over the long term for $N_{i}$ and $V_{i}$ users, respectively. These figures are mathematically derived in [25] for proportional fair scheduling as follows:

$$
\begin{aligned}
\mathrm{R}_{N j}^{i} & =\frac{\mathrm{G}\left(N_{i}^{N}(\alpha)\right) \cdot r_{N j}^{i}}{N_{i}^{N}(\alpha)}, \\
\mathrm{R}_{V j}^{i} & =\frac{\mathrm{G}\left(N_{i}^{V}(\alpha)\right) \cdot r_{V j}^{i}}{N_{i}^{V}(\alpha)}
\end{aligned}
$$

while $r_{N j}^{i}$ and $r_{V j}^{i}$ are the link data rates for nonvictim and victim users respectively. Maximizing (4) is NP-hard to solve therefore the authors in [14] and in this work, we have considered DP solution. The DP solves this problem with complexity reduction from $O\left(2^{k_{i}}\right)$ to $O\left(k_{i}^{2}\right)$, where $k_{i}$ is the number of users per $S B S_{i}$, while 2 represents the subsets of small users to be classified. To conclude, the selection criteria of the particular small-cell user as $N_{i}$ or $V_{i}$ in $S B S_{i}$ are based on maximizing the aggregated utility function $U_{i}^{\text {tot }}(\alpha)$. The maximized $U_{i}^{\text {tot }}(\alpha)$ for a given value of muting ratio $\alpha$ is defined by

$$
U_{i_{\text {max }}}(N, V \mid \alpha)=\max \left\{U_{i}(N, V \mid \alpha)\right\}
$$

In other words, each new added user can be either $N_{i}$ or $V_{i}$ and his/her associated link rate is added to both subutility $U_{i}^{N}(\alpha)$ and $U_{i}^{V}(\alpha)$ to searches for $U_{i \text { max }}(N, V \mid \alpha)$ across possible subutility functions, which in turn determines the maximal utility function. Based on what is above referred and (3), (4), and (5), $U_{\text {imax }}(N, V \mid \alpha)$ is given by (8) and illustrated in step 7 in the DP Victim's selection stage pseudocode:

$$
\begin{aligned}
& U_{i \max }(N, V \mid \alpha)=\max \left\{\left\{U_{i \max }(N-1, V \mid \alpha)\right.\right. \\
& \left.+\log _{2}\left[(1-\alpha) \cdot r_{i}^{N} \cdot \frac{(N-1)^{N-1} \cdot G\left(N_{i}^{N}(\alpha)\right)^{N}}{N^{N} \cdot G\left(N_{i}^{N}(\alpha)-1\right)^{N-1}}\right]\right\}, \\
& \left\{U_{i_{\text {max }}}(N, V-1 \mid \alpha)\right. \\
& \left.\left.+\log _{2}\left[(\alpha) \cdot r_{i}^{V} \cdot \frac{(V-1)^{V-1} \cdot G\left(N_{i}^{V}(\alpha)\right)^{V}}{V^{V} \cdot G\left(N_{i}^{V}(\alpha)-1\right)^{V-1}}\right]\right\}\right\}
\end{aligned}
$$

By stating the final $N_{i}$ and $V_{i}$ in each $S B S_{i}$ that realize $U_{i_{\text {max }}}(N, V \mid \alpha)$. The RIE in C-RAN centre (BBU pool) will be able to label every small-cell user as victim and nonvictim in each picocell individually as clarified in the pseudocode of the $\boldsymbol{D P}$ victim selection Algorithm 1 (step 3 to step 12).

3.2.2. DP-DAP Methodology and Principles. On the contrary to [14], the proposed ABS-based scheme does not rely on applying $\mathrm{ABS}$ on macrousers. The authors in [14] have obtained the optimum ABS density by computing maximal utility for each small-cell user and the utility function for the same value of $\alpha$ (muting ratio) for the macrocell users and then calculate the overall system utility function for all possible values of $\alpha$. Based on comparison process, the optimum 


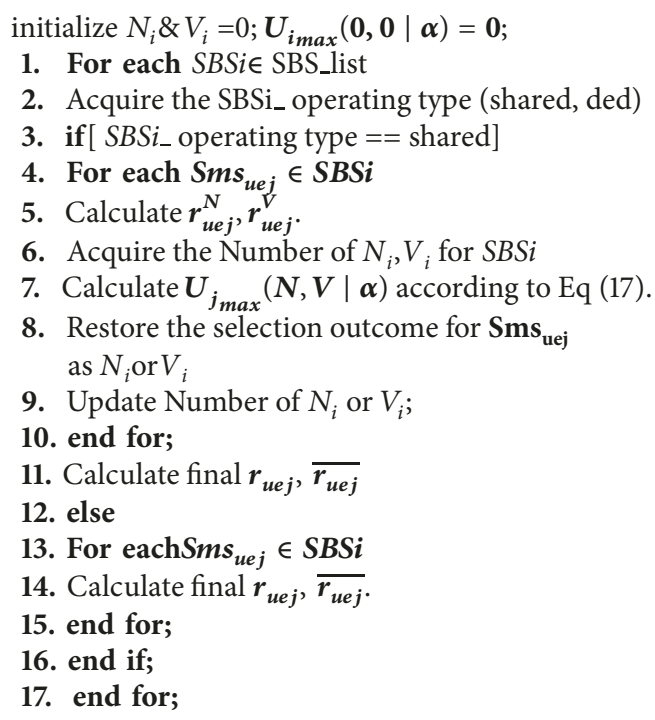

Algorithm 1: DP Victim’s selection stage.

value of the ABS density for synchronous $\mathrm{ABSF}$ is obtained. In classic ABSF deployment scenarios, the decision to whether to configure ABSF in a network includes trade-off factors between capacity gain due to range expansion of the small cells when we apply power bias and the capacity loss due to inability to allocate resources for macrocell users in the ABSs of the interfering MBS [26]. In literature for simplicity, MBSs work only in non-ABS and mute totally in ABSF. However, SBSs work in all subframes and victim UEs are preferentially scheduled in ABS subframes. Another assumption is that all Home eNBs (HeNBs) or SBSs deployed in the macrocell area can execute a synchronization procedure to synchronize with the MBS. The MBS triggers the aggressor HeNBs through the backhaul link to operate in the ABSF mode, with a chosen ABSF pattern.

3.2.3. Macrocell Sectorization in DP-DAP. The authors in $[14,27]$ have assumed that the macrocell users by default are not scheduled in muted subframes in order to mitigate the severity of interference on $S B S$ victim users which in turn will cause loss in capacity for macrocell users. Moreover, there are other factors in terms of practicality when applying fixed ABS pattern; for instance, at low system load, the probability of users experiencing cochannel interference is low and there is no need for ABSs. Another factor is when the ABSs are configured, the number of macrousers handed over to SBS when applying the Cell Range Expansion (CRE) will affect the number of ABSs required. To address the above-mentioned issues, this study applies a new concept of sectorizing the vicinity of the wide macroregion into variable number of sectors depending on the ABS density. ABS density (referred to as $\alpha$ ) plays a key role on how many sectors the macrovicinity is sliced. For instance, when $\alpha=0.4$, then there are two ABS patterns that result in two sectors in the macroarea.
1. Acquire total number of sub-frames $\boldsymbol{s} \boldsymbol{b} \boldsymbol{f r m e}_{\boldsymbol{t o t}}, \boldsymbol{\alpha}$, NuofMacropersbfrme, SBS_list, MMS_list, Sms_list

2. for each subframe f $_{i} \in \boldsymbol{s b}$ frme $_{\text {tot }}$

3. Acquire the associated sector and $S B S s$;

4. Apply ABSF-pattern for $S B S s$ in the associated sec

5. While counter $<$ NuofMacropersbfrme

6. Index $=$ index +1 ;

7. if $\left[M M S_{j}\right.$ (index)'s sector != the associated sector]

8. $M M S_{j}$ is allocated resources;

9. Mark $\boldsymbol{M} \boldsymbol{M} \boldsymbol{S}_{\mathbf{j}}$ as scheduled;

10. Counter $=$ Counter +1 ;

11. end if;

12. end while;

13. Counter $=0$;

14. end for;

Algorithm 2: DP-DAP for macrousers.

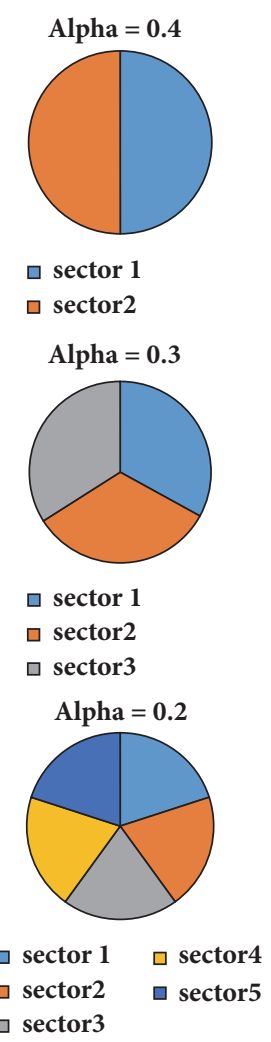

Figure 4: Mapping between Alpha and the cell layout.

Figure 4 depicts the mapping between $\alpha$ and the layout of cell in terms of the number of sectors. This scheme is running on subframe basis and termed (DP-Diverse ABSF Pattern) referred to as DP-DAP. For instance, at a specific ABS subframe $X$, a SBS's victim user are allocated resources in subframe $X$, such that only macrousers that reside in the same sector as $S B S$ will not be scheduled on that subframe. Nevertheless, macrousers who are distributed in other sectors are allocated in subframe $X$ (steps 7 and 8 in Algorithm 2). This can be realized practically by today antenna's technology 

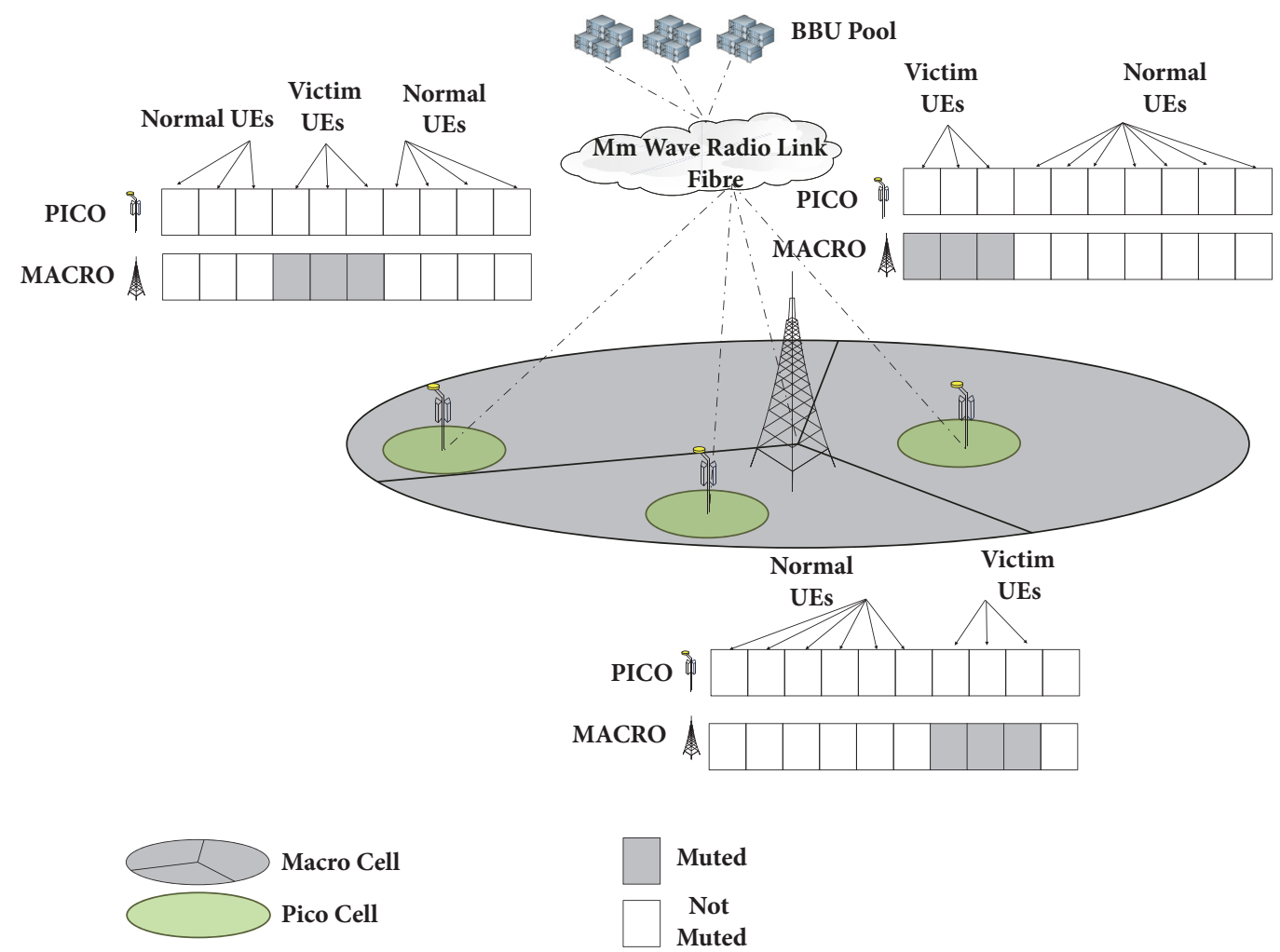

Figure 5: Diverse-ABSF patterns in sectorized H-CRAN.

(beam forming, directional antenna, etc.). The pseudocode associated with the above-mentioned method is shown in Algorithm 2.

Figure 5 demonstrates a macrocell with small cells scattered in its vicinity, all connected to the BBU pool in the data centre. ABS density in this example is $3 / 10$ and macroregion is sliced into three different areas. Small cells in different sectors have different ABS patterns; this allow macrousers in other sectors to utilize the muted subframes which will ease the capacity loss of legacy ABS.

\section{System Model}

4.1. Network Model. In this study, a cellular architecture of cloud cooperated HetNet is deployed to operate in a single band HetNet. We consider two-tier C-RAN HetNet composed of (1) the first tier formed by the macrocellular BS with RRH capabilities only, while the subsequent tiers correspond to small cellular RRHs (picocells in this study) with antennas located at remote sites deploying LTE in our study, assuming that the RRHs connect to the BBU through ideal optical fibre; (2) BBU pool with enhanced collaborative functionalities between different tiers sites; (3) high bandwidth fronthaul links between the RRHs and the BBU pool. We assume that all tiers are connected to a cloud data centre, which hosts the BBUs pool. A front RF and simple processing functionalities are implemented in RRHs, while the rest of the baseband processing procedures and upper layers are executed jointly in the BBU pool. The spatial configuration of the RRHs in the second tier is modelled using uniform distribution in the considered macrocell area (tier 1 cell). Figure 6 illustrates a multitiers C-RAN HetNet as a coexistence scenario for one MBS and multiple SBSs that represent the pico and femto in HetNet; however, this study considers only picocells case. The coverage of each cell depends on its type (i.e., an MBS or an SBS). The number of users associated with each SBS varies as discussed in Section 4.3. The BBU pool maintains regular signalling with the MBS and SBS to collect information about user's conditions (such as SINR levels). In this proposal, the SBS is assumed to be able to operate in the shared partition of the spectrum along with the MBS or the dedicated part. Both partitions are allocated based on the output of our proposed algorithm which is executed at the RIE in the BBU pool. The downlink transmission is considered in which the BBU pool sends individual data flows to users via RRHs.

4.2. Wireless Channel Model. In this study, a 3D 3GPP/ITU channel model proposed in a previous work [28] is implemented which considers both propagation in the azimuth and elevation planes. The 3D ITU model accounts for large scale fading by considering path loss and shadowing effects and small scale fading through detailed modelling of the Multipath Components (MPCs). The large scale parameters (LSPs) used in this model are based on 3D ray tracing predictions, providing information on the amplitude, phase, time delay, Angle of Arrival (AoA), and Angle of Departure (AoD) in elevation and azimuth domains. Readers are referred to our previous work in [28] for detailed explanation of the 

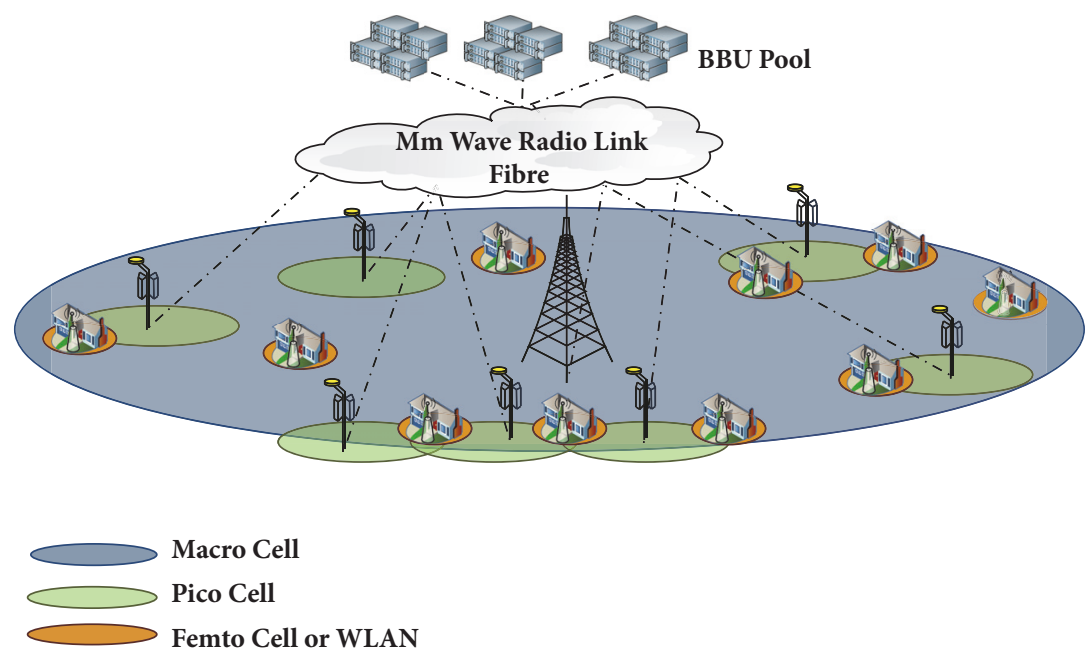

FIGURE 6: H-CRAN architecture[22].

TABLE 2: Definition of channel matrix variables.

\begin{tabular}{ll}
\hline Parameter & Description \\
\hline$P_{n}$ & Cluster power \\
\hline$E_{r x, u, V}, E_{r x, u, H}$ & Vertical and horizontal polarization components of receive and transmit antenna radiation patterns \\
$E_{t x, s, V}, E_{t x, s, H}$ & Received co-polarized signal \\
\hline Superscripts VV and & Received cross-polarized signal \\
HH & Initial phases of a ray $m$ in cluster $n$ \\
\hline Superscripts VH and & Cross-polarization power ratio \\
\hline$\Phi_{n, m}^{V V}, \Phi_{n, m}^{V H}, \Phi_{n, m}^{H V}, \Phi_{n, m}^{H H}$ & Tx and Rx unit vector defined by equations $(3)$ and $(4)$ \\
\hline$\kappa$ & Coordinate of Tx and Rx MIMO antenna elements $j$ and $i$ \\
\hline$\Psi_{n, m}^{T x}, \Psi_{n, m}^{R x}$ & Wavelength of the carrier frequency \\
\hline$\Omega_{j}^{T x}, \Omega_{i}^{R x}$ & UE speed in the direction of $(\theta, \phi)$ \\
\hline$\lambda_{0}$ &
\end{tabular}

generation of our implemented 3D channel statistics and the modelling of the related LSPs. The system was modelled at a carrier frequency of $2.6 \mathrm{GHz}$, and the implemented path loss models for nonline-of-sight condition, Bristol, United Kingdom, is shown in

$$
P L_{N L O S}=39 \log _{10}(d)+25
$$

The mathematical derivation of the time variant 3D channel matrix is expressed in (10), with description of the terms that compose the channel matrix is defined in Table 2. Detailed description of each of the channel matrix components is provided in [29]. Please note that in (10), the angles of departure and arrival for each multipath ray in the elevation and azimuth domains are referred to as $(\theta, \phi)_{n, m, A o D}$ and $(\theta, \phi)_{n, m, A o A}$, respectively. In addition, in (10), the subscripts $u$ refer to receive antenna element, $s$ for transmit antenna element, $n$ for ray cluster number, and $m$ for a subpath ray in the cluster. Equation (10) represents the time variant channel of a MIMO system in general between any receiving antenna $u$ and transmit antenna s. Equation (10) also illustrates the entities that constitute the channel coefficients. Therefore, the final channel matrix is a spatial convolution of the power of each MPC cluster with the receive and transmit antenna radiation patterns, taking into account the placement of the antenna elements on xyz coordinates and the Doppler shift in case of mobility as illustrated in (10). In case of polarized antenna patterns, a $2 \times 2$ polarization matrix is added. The receiving and transmit coordinate vectors provided in (10) are expressed in detail in (11) and (12). Please refer to [29] for further details of the $3 \mathrm{GPP} / \mathrm{ITU}$ channel modelling. 


$$
\begin{aligned}
& H_{u, s, n(t)=\sqrt{P_{n}}}{ }^{m=M}\left[\begin{array}{l}
E_{t x, s, V}\left((\theta, \phi)_{n, m, A O A}\right) \\
E_{t x, s, H}\left((\theta, \phi)_{n, m, A O A}\right)
\end{array}\right]^{T} \\
& *\left[\begin{array}{cc}
\exp \left(j \Phi_{n, m}{ }^{v v}\right) & \sqrt{\mathrm{K}_{n, m}} \exp \left(j \Phi_{n, m}{ }^{v h}\right) \\
\sqrt{\mathrm{K}_{n, m}} \exp \left(j \Phi_{n, m}{ }^{h v}\right) & \exp \left(j \Phi_{n, m}{ }^{h h}\right)
\end{array}\right] \\
& *\left[\begin{array}{l}
E_{r x, u, V}\left((\theta, \phi)_{n, m, A O D}\right) \\
E_{r x, u, H}\left((\theta, \phi)_{n, m, A O D}\right)
\end{array}\right]^{T} \\
& * \exp \left(j \frac{2 \pi}{\lambda_{0}} \Omega_{i}^{T x} \cdot \Psi_{n, m}^{T x}\right) \\
& * \exp \left(j \frac{2 \pi}{\lambda_{0}} \Omega_{i}^{R x} \cdot \Psi_{n, m}^{R x}\right) \\
& * \exp \left(j 2 \pi v_{n, m} t\right)
\end{aligned}
$$

$$
\begin{aligned}
& \Psi_{n, m}^{T x}=\left[\begin{array}{lll}
\cos \phi_{n, m}^{A o D} \cos \theta_{n, m}^{A o D}, & \sin \phi_{n, m}^{A o D} \cos \theta_{n, m}^{A o D}, & \sin \theta_{n, m}^{A o D}
\end{array}\right]^{T} \\
& \Psi_{n, m}^{R x}=\left[\begin{array}{lll}
\cos \phi_{n, m}^{A o A} \cos \theta_{n, m}^{A o A}, & \sin \phi_{n, m}^{A o A} \cos \theta_{n, m}^{A o A}, & \sin \theta_{n, m}^{A o A}
\end{array}\right]^{T}
\end{aligned}
$$

4.3. Simulation Setup. Consider the HetNet network model presented previously, a total number of 200 users are uniformly distributed in the entire area of the macrocell. Therefore, each user is associated with the BS that has the highest received power. This implies different SBSs have different number of associated users. The simulation parameters are provided in Table 3.

\section{Analysis of Simulation Results}

5.1. Operating DP-DAP with UWPA. The previously proposed UWPA allows high number of small cells to utilize the same resources as MBS while considering the intertier interference mitigation. However, by observing Figure 8, small-cell user's SINR, it can be noticed that their SINR is lower than other schemes although the end throughput is higher. The higher throughput obtained in our methodology is due to the greater shared spectrum utilized by small cell users. The hybrid scheme is the extended version of UWPA. It involves UWPA as the first step in frequency or resource division; however it inserts a time division mechanism based on the proposed ABSF with vicinity sectorizing (DP-DAP). After SBSs are identified in terms of resources as shared or dedicated, the stage of DP Victim's selection is applied to mark the small-cell users as victim or nonvictim. Time division on shared SBSs only that allows allocating victim and normal users in different subframes are employed as the last stage. The macrousers in the meantime are mapped to their associated sectors and subframes for resource allocation as described in the diverse-ABSF Pattern mechanism and the associated pseudocode algorithm is shown in Algorithm 2: DP-DAP for macrousers.

In order to demonstrate the performance gain of the joint DP-DAP proposed in this article and the previously proposed UWPA, the results are benchmarked against state-of-the-art methods as discussed below.

The criteria of victim small-cell users selection in literature has counted on many metrics such as considering all certain SBS's small-cell users as victim users that are scheduled only in ABSs while all macrousers are scheduled in non-ABS [27]. Another metric is based on considering the small-cell users located in the CRE when applied as victim users and small-cell users in the main vicinity before applying CRE as nonvictim users, this metric is termed as CRE-based or CRE. The CRE technique allows the users to associate with the BS with lower SINR [30]. It is considered as an effective mechanism for traffic offloading to increase the total system capacity. The CRE has been used in literature to expand the small-cell range virtually by adding a bias to the small-cell received power, instead of increasing the transmit power of the small-cell BS; this improves cell-edge throughput and overall network throughput. Many research studies have focused on intercell interference coordination in CRE; this is due to the fact that many macro-BS strong power harms the expanded region users that uses biases to connect to lower power BS, and therefore difference mechanisms 

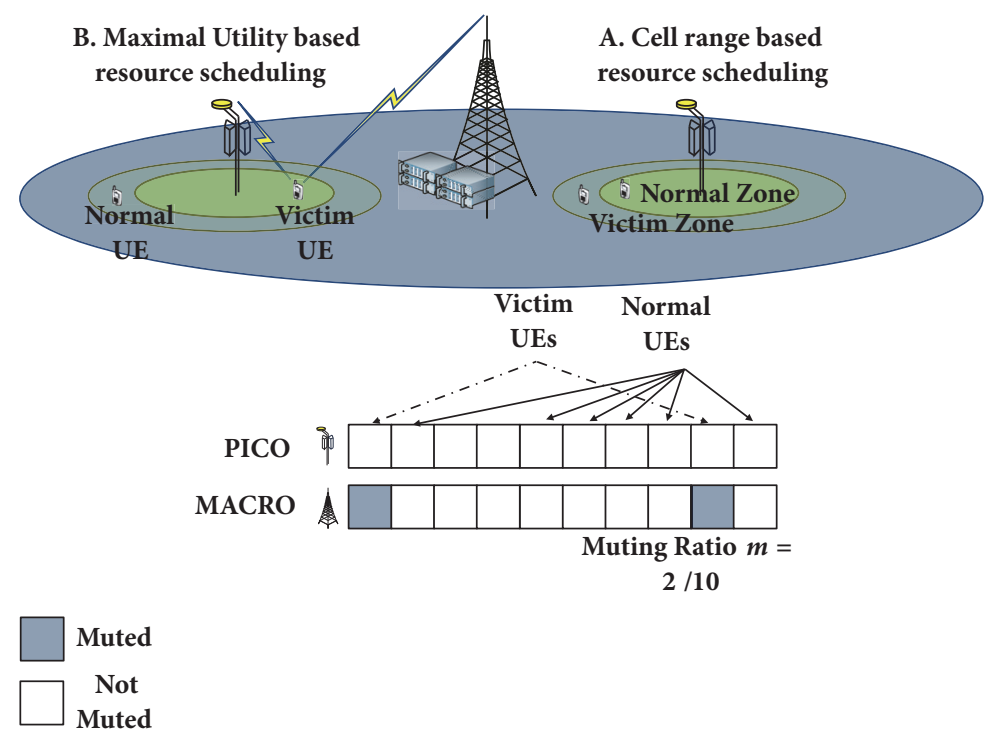

Figure 7: Cell range and maximal utility based resources allocation in H-CRAN.

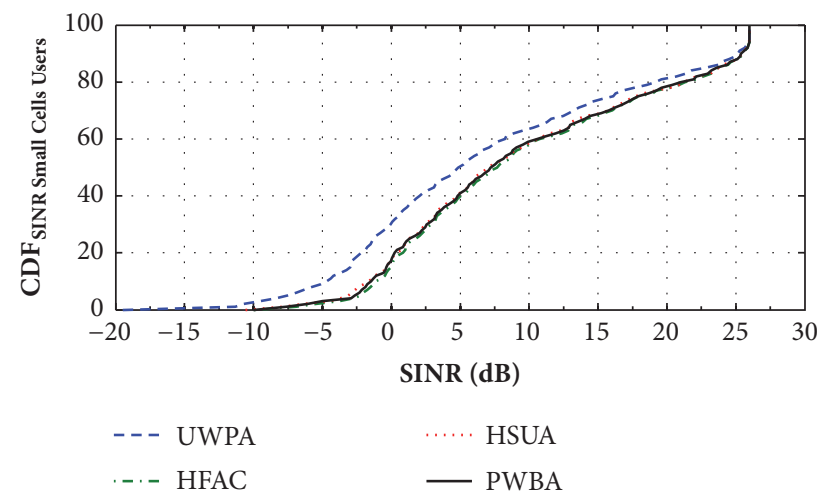

FIGURE 8: SINR of small-cell users in UWPA, HFAC, HSUA, and PWBA-CDF.

have been proposed to calculate the optimum bias values.

This study benchmarks against the above-referred metrics in its time division section. Figure 7 illustrates the difference between utility-maximization based scheme used in this study and CRE-based. It shows that victim user can be resided in the inner vicinity (before applying CRE) as well as outer vicinity (when applying CRE). Therefore, the proposed framework is compared against three main other schemes: AS (where all small-cell users are scheduled in the ABSs), CREbased (where both schemes will be running on top of UWPA), and UWPA-based only. UWPA-based involves the outcome from the first stage of this hybrid work (UWPA resources partition). This framework assumes an ABS pattern period as $10 \mathrm{~ms}$ (10-subframe duration). The aim of the study is SINR enhancement for small-cell users.

5.2. Small User's SINR Improvement. This section presents the SINR performance of the small-cell users. The SINR is
TABLE 3: Simulation parameters [the table is reproduced from $\mathrm{R}$. Almesaeed et al. (2017) (under the Creative Commons Attribution License/public domain)].

\begin{tabular}{lc}
\hline Parameter & Values \\
\hline Macro cell radius & $1500 \mathrm{~m}$ \\
\hline MBS Transmission Power & $49 \mathrm{dBm}$ \\
\hline Total number of users & 200 \\
\hline SBS transmission power & $20 \mathrm{dBm}$ \\
\hline Number of pico cells & 10 \\
\hline Bandwidth & $20 \mathrm{MHz}$ \\
\hline Antenna type & Isotropic \\
\hline Carrier frequencies & $2.6 \mathrm{GHz}$ \\
\hline Wireless channel model & Extended 3D 3GPP/ITU \\
\hline Propagation condition & NLOS, Urban \\
\hline Number of network realizations & 3 \\
\hline
\end{tabular}

calculated according to the following equation:

$$
\operatorname{SINR}_{U_{i}, B S_{j}}=\frac{P_{j}\left(L_{U_{i}, B S_{j}}\right)^{-1}}{N_{0}+\sum_{x}\left(B S_{x}\right) P_{x}\left(L_{U_{i}, B S_{x}}\right)^{-1}}
$$

where $S I N R_{U_{i}, B S_{j}}$ is the SINR at user $\left(U_{i}\right)$ associated with BS $\left(B S_{j}\right) . P_{j}$ is the transmission power of the associated BS $\left(B S_{j}\right), L_{U_{i}, B S_{j}}$ represents the power loss introduced by the large scale fading due to path loss and small scale fading due to multipath propagation (the small scale fading is modelled by the channel matrix computed in Section 4.2), between the user $\left(U_{i}\right)$ and associated BS $\left(B S_{j}\right)$, and $N_{0}$ is the noise power at the UE defined by (14). $B S_{x}$ represents the list of interfering BSs with $B S_{j}, P_{x}$ is the transmission power of the interfering BS $x$, and $L_{U_{i}, B S_{x}}$ is the power loss as mentioned previously 


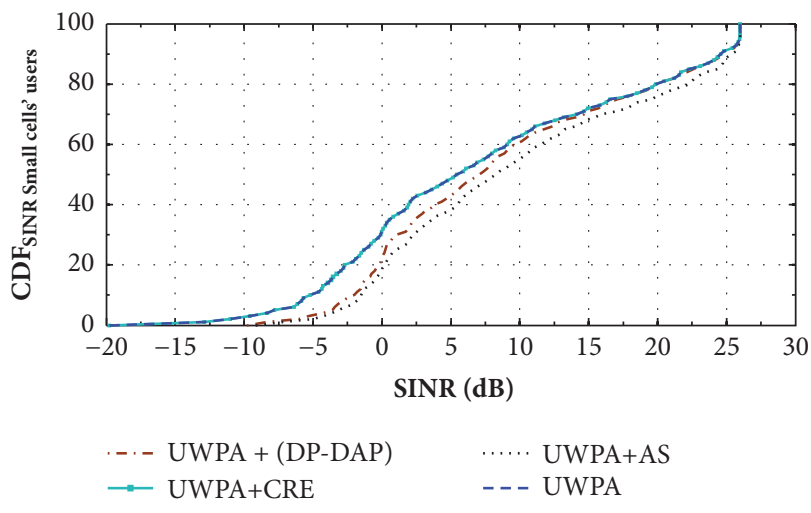

(a) $\mathrm{BIAS}=0 \mathrm{~dB}$

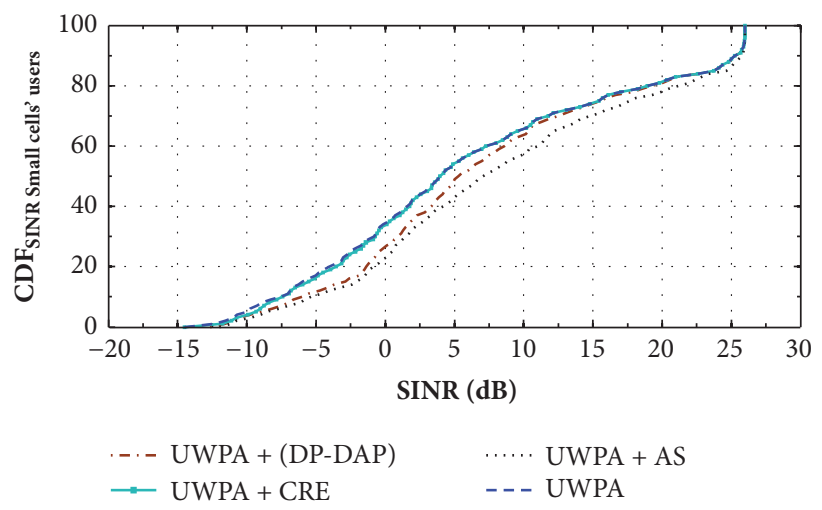

(b) $\mathrm{BIAS}=3 \mathrm{~dB}$

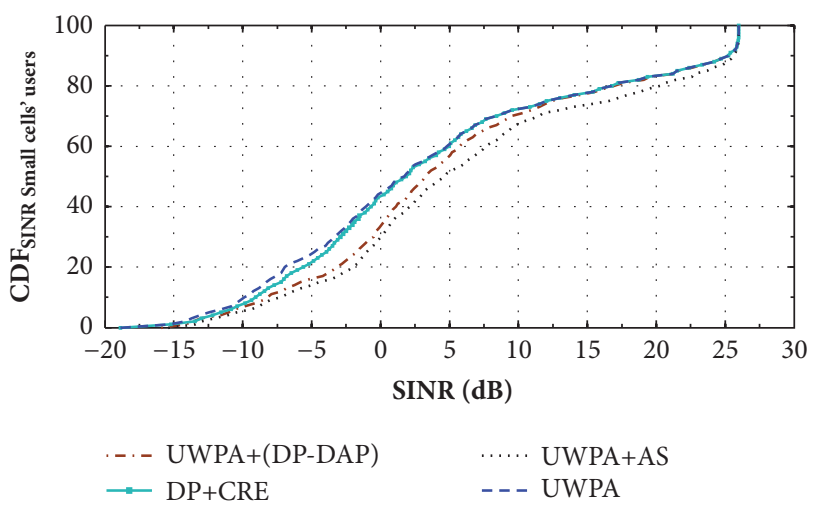

(c) BIAS $=6 \mathrm{~dB}$

FIGURE 9: SINR of small-cell users in UWPA, UWPA+AS, UWPA+CRE, and UWPA+(DP-DAP)-CDF.

between the interfering $B S_{x}$ and user $U_{i}$. The white noise $N_{0}$ is calculated as

$$
N_{0}=K T B(d B m)+N F(d B)
$$

In (14), $K$ is Boltzmann's constant, $T$ is the temperature in Kelvin, $B$ is the effective bandwidth $(90 \%$ of the total bandwidth), and NF is the noise figure. In this article, the LTE-Advanced Bandwidth of $10 \mathrm{MHz}$ is considered and for the UE, $T=15^{\circ} \mathrm{C}$ (288 Kelvin), and $N F=9 \mathrm{~dB}$ [31].

Figure 8 illustrates small-cell user's SINR with UWPA as compared with previous schemes presented in Section 3.1.2. The figure compares $H F A C, H S U A$, and $P W B A$ against UWPA. 50\% of small-cell users experience less than $8.247 \mathrm{~dB}$ in HFAC, $7.94 \mathrm{~dB}$ in HSUA and $7.967 \mathrm{~dB}$ in PWBA. While 50\% of small-cell users experience less than $5.415 \mathrm{~dB}$ in UWPA. Figure 9 highlights the improvement occurred in small-cell user's SINRs due to the use of $D P-D A P$.

Figures 9(a), 9(b), and 9(c) cover the case where there is (no power, $3 \mathrm{~dB}$ bias, and $6 \mathrm{~dB}$ bias) applied, respectively. The other baselines to compare against are (UWPA $+A S)$ and $(U W P A+C R E)$ as well as UWPA only. It can be observed from Figures 9(a) and 9(b) that the SINR of small-cell users in UWPA, (UWPA+AS), $(U W P A+C R E)$, and $(U W P A+\mathrm{DP}-\mathrm{DAP})$, applying $(U W P A+\mathrm{CRE})$ with no or small bias value will not lead to any enhancement. It is intuitive that $(U W P A+\mathrm{CRE})$ with zero bias is considered the same as UWPA, Therefore, no improvement is expected in the first place and that is why their associated curves match in Figure 9. Moreover, Figure 9(b) depicts little deviation noticed from Figure 9(a) between UWPA and (UWPA+CRE). However, Figure 9(c) affirms that applying higher bias (6 dB for instance) leads to improvement over the original UWPA. Nevertheless, the improvement is minor compared to other applied schemes (UWPA+AS, UWPA+(DP-DAP)). This due to the fact that with small bias values the number of victim users is small compared to the total number of users in the case with no bias applied. This implies that their SINR improvement will not impact the overall cell SINR. SINR improvement in $(U W P A+(D P-D A P))$ can be noticed as $50 \%$ of small-cell users experience less than $3.235 \mathrm{~dB}$ compared to $55 \%$ for the same SINR in UWPA (5\% enhancement) in Figure 9(c). Similar observations are taken from Figures 9(a) and 9(b). Previous schemes are worse than (UWPA $+A S$ ) because it is an orthogonal reuse case; thus there is no interference from $M B S$ towards SBS's users.

5.3. Macrousers Performance. As referred in Section 3.2.3, it is expected that slicing of macroregion into sectors is more efficient in terms of capacity loss for macrousers than classic ABSF that considers a RR scheduler. Therefore, two metrics are assessed in our study, the first metric is macrouser's average throughput while the second one is a statistical measure to record how many times an average macrouser is scheduled and named as scheduling time. 
TABLE 4: Calculated throughput (average UE rate in bps/UE) for macrousers.

\begin{tabular}{lccc}
\hline Alpha & \multicolumn{3}{c}{ Bias } \\
& 0 & 3 & 6 \\
\hline 0.2 & $4.4 e 5 / 3.6 \mathbf{e} 5$ & $4.9 e 5 / 3.9 \mathbf{e} 5$ & $5 e 5 / 4 \mathbf{e} 5$ \\
\hline 0.3 & $4.3 e 5 / 3 \mathbf{e} 5$ & $4.7 e 5 / 3 \mathbf{e} 5$ & $5.2 e 5 / 3.7 \mathbf{e} 5$ \\
\hline 0.4 & $4.5 e 5 / 2.7 \mathbf{e} 5$ & $4.5 e 5 / 2.8 \mathbf{e} 5$ & $5.2 e 5 / 3.2 \mathbf{e} 5$ \\
\hline
\end{tabular}

TABLE 5: Number of scheduling times for macrousers.

\begin{tabular}{lccc}
\hline Alpha & \multicolumn{3}{c}{ Bias } \\
& 0 & 3 & 6 \\
\hline 0.2 & $144 / \mathbf{1 1 7}$ & $159 / \mathbf{1 2 9}$ & $172 / \mathbf{1 4 0}$ \\
\hline 0.3 & $145 / \mathbf{1 0 3}$ & $156 / \mathbf{1 1 0}$ & $172 / \mathbf{1 2 3}$ \\
\hline 0.4 & $145 / \mathbf{8 7}$ & $158 / \mathbf{9 6}$ & $170 / \mathbf{1 0 4}$ \\
\hline
\end{tabular}

Tables 4 and 5 show the results for both metrics respectively. The results are calculated for both schemes $(U W P A+\mathrm{DP}-\mathrm{DAP}$ (presented in italic)) and (UWPA+ classic $\mathrm{ABSF}$ (bold)) considering different bias and $\alpha$ values. It can be noticed from table that $\alpha$ value has no impact on scheduling times for macrousers as they are scheduled in muted ABSs too. However, increasing $\alpha$ in classic ABSF reduces scheduling times as they are inversely proportional. This intuitively leads to lower associated throughput. Increasing the power bias has an effect as it allows more original macrousers to associate with SBSs which reduces the total number of macrousers in the macroregion; this in turn increases the scheduling times as can be observed from Table 5. It is worth mentioning that the macrothroughput presented in this section is calculated according to (15), and it presents the error-free data throughput for the LTE system, calculated based on the number of scheduling times and number of resources assigned for each user as in

$$
\text { Thr_macro }=\frac{T_{\text {sch }} * R B U * 12 * 7 * 2 * R * k_{\text {mod }}}{S_{\text {time }}},
$$

where the Thr_macro is the error-free data rate (specific for LTE system and particular bandwidth), $R B U$ is the number of resource blocks assigned for particular user, $R$ is coding rate, $k_{\text {mod }}$ is the modulation order, and $S_{\text {time }}$ is the simulation time. The numbers 12,7 , and 2 refer to the number of carriers per resource block, number of OFDM symbols per slot, and number of slots per subframe respectively. The proposed $(U W P A+D P-D A P)$ scheme shows an enhancement in terms of calculated throughput and number of scheduling times against (UWPA + Classic ABSF).

The numerical results in Tables 4 and 5 highlight the performance enhancement for all cases, for instance, when $\alpha$ $=0.3$ and Power Bias $=3 \mathrm{~dB},(U W P A+D P-D A P)$ achieves $56 \%$ improvement in average user data rate and $41 \%$ in number of scheduling times.

5.4. Small Users' throughput Enhancement. The design of DP$D A P$ on top of UWPA is anticipated to make the smallcell users experience higher throughput since it proves improvement on their SINR as mentioned in Section 5.2. The small-cell user throughput is calculated according to Shannon (Ergodic) Capacity in

$$
C=B * \log _{2}(1+\gamma)
$$

$B$ is the volume of the spectrum available for that particular cell and $\gamma$ is the received SINR. In order to demonstrate the enhancement introduced by the DP-DAP technique, the comparison is performed against $(U W P A+A S)$, $(U W P A+C R E)$, and small ell user's throughput just after applying UWPA only. The performance is evaluated for the case of $3 \mathrm{~dB}$ cell range bias and three different $\alpha$ values (0.2, 0.3 , and 0.4).

Figures 10(a), 10(b), and 10(c) show the corresponding average throughput CDF curves in regard with $(\alpha=0.4,0.3$, and 0.2$)$, respectively. It is observed that $(U W P A+D P-D A P)$ has achieved improvement over UWPA throughput for all $\alpha$ values. For example, the hybrid scheme $(U W P A+D P-D A P)$ achieves $6 \%$ improvement in number of users achieving throughput higher than 2 Mbps against UWPA only. Applying CRE on top of UWPA has no change to UWPA only apart from slight diversion at higher rates. UWPA only is even better than applying $(U W P A+A S)$ as the latter underperforms by $22 \%$ in the number of users that experience throughput greater than $2 \mathrm{Mbps}$. The DP-DAP technique divides smallcell users into victim and nonvictim users. Therefore, to make the comparison fair with UWPA results, this study considers measuring average throughput for victim and nonvictim users. Nonetheless average throughput is calculated by the following:

$$
\overline{r_{i j}}=\frac{r_{i j}}{K_{i}}
$$

where $K_{i}$ is the total number of small users in particular cell. Instead, $\overline{r_{i j}}$ is calculated by dividing $r_{i j}$ over the total number of small-cell users of the same type (victim or nonvictim) in particular cell. Without loss of generality, the average throughput improvement is due to the victim smallcell users' SINR improvement which leads to better data rate. That is beside the fact that normal small-cell users average throughput in any SBS has not changed because of dedicating other resources for victim small-cell users. Figures 11(a), 11(b), 11(c), 12(a), 12(b), and 12(c) depict the average throughput for victim and nonvictim small-cell users, respectively, for $\alpha$ $=0.4,0.3$, and 0.2 , respectively, again. For $\alpha=0.4$, nonvictim small-cell users have the highest improvement against UWPA compared with other $\alpha$ values $(0.3,0.2)$. For instance, $\alpha=0.4$ results in $50 \%$ of users with less than $3.71 \mathrm{Mbps}$ as compared to 3.213 Mbps for $\alpha=0.3$ and 2.304 Mbps for $\alpha=0.2$.

Conversely, victim small-cell users show the highest improvement against their peers in UWPA when $\alpha=0.2$ as noted from Figure 12(c). This is because when $\alpha$ equals 0.2 , victim small-cell users have less resources than the case of $\alpha=0.3$ and 0.4 , but the total number of victim small-cell users in a particular cell is less (when $\alpha=0.2$ ) according to the initial classification process (DP Victim's selection stage). Therefore, the victim users have a greater chance to be allocated resources. 


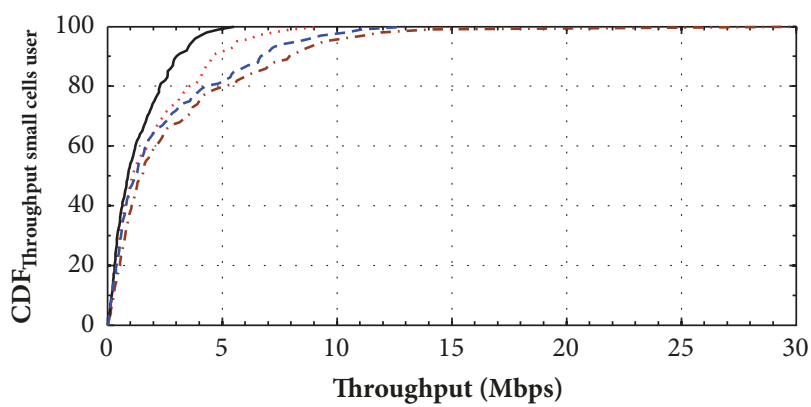

$\begin{array}{ll}-\cdots & \text { UWPA + (DP-DAP) UWPA+AS } \\ \cdots . . \text { UWPA+CRE } & --- \text { UWPA }\end{array}$

(a) $\alpha=0.4$

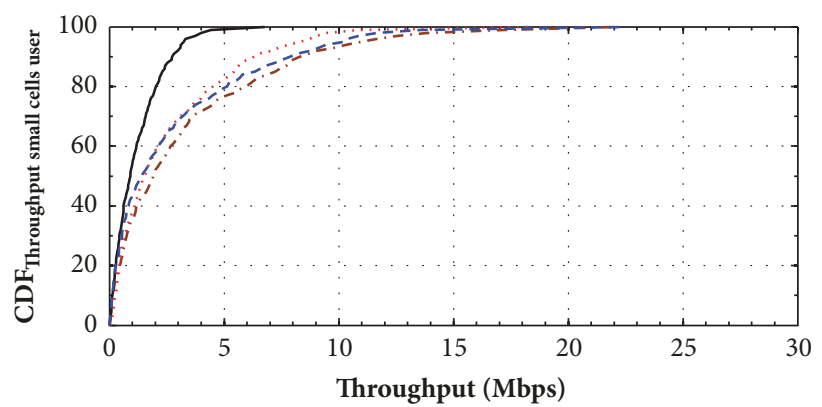

... UWPA + (DP-DAP) - UWPA+AS

(b) $\alpha=0.3$
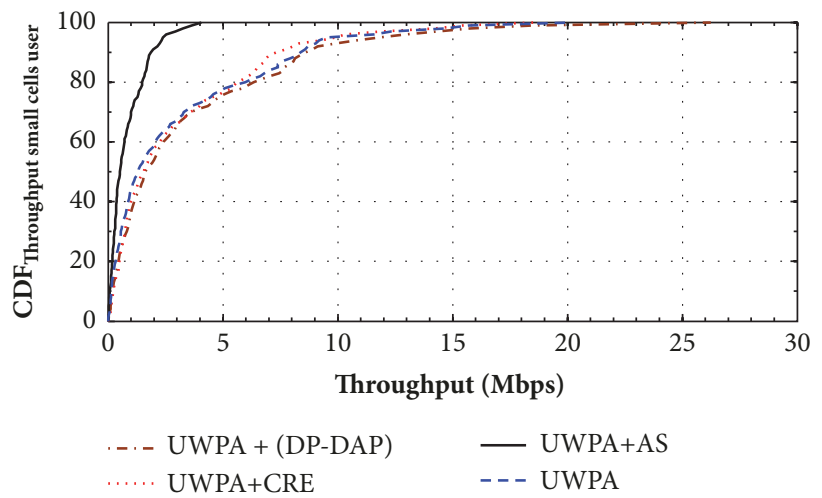

(c) $\alpha=0.2$

Figure 10: Small users' average throughput for different Alpha values (CDF).
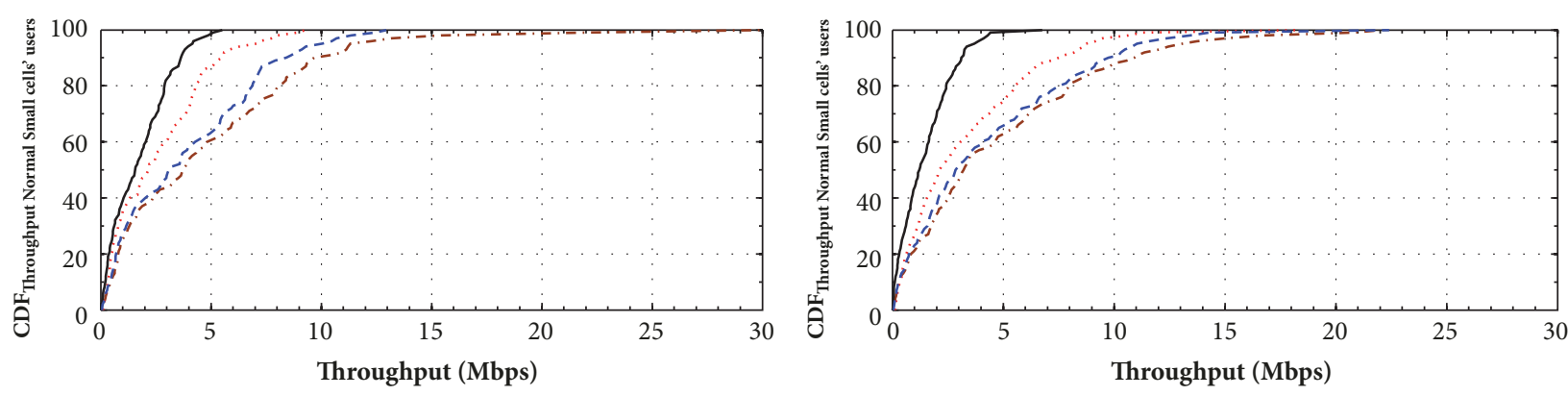

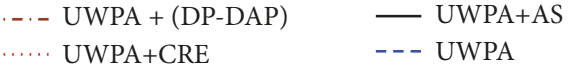

(a) $\alpha=0.4$

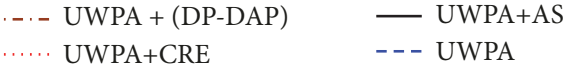

(b) $\alpha=0.3$

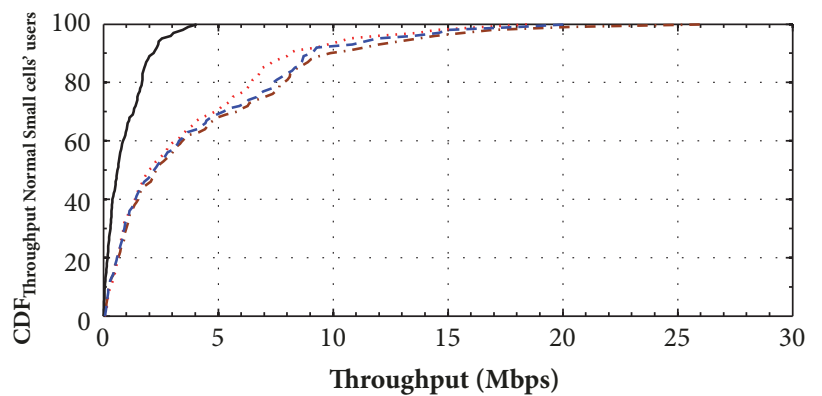

$\begin{array}{ll}-\cdots & \text { UWPA + (DP-DAP) } \\ \text {-... UWPA+CRE } & \text { UWPA+AS }\end{array}$

(c) $\alpha=0.2$

FIGURE 11: Average throughput for nonvictim small cells' users (CDF). 


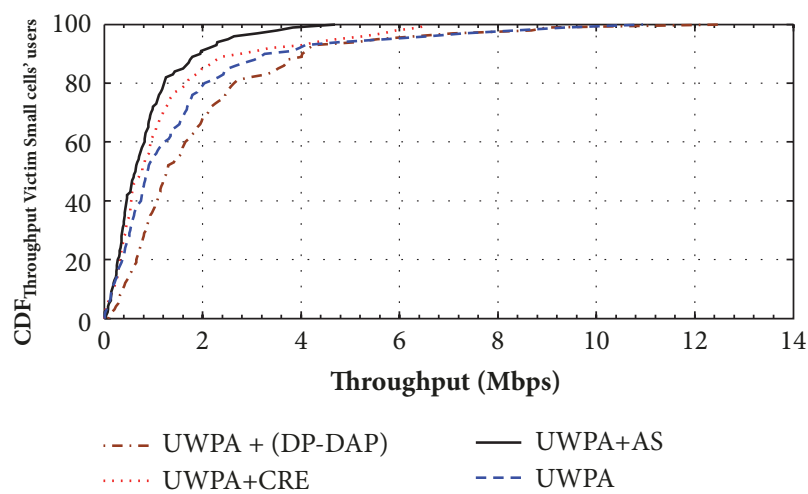

(a) $\alpha=0.4$

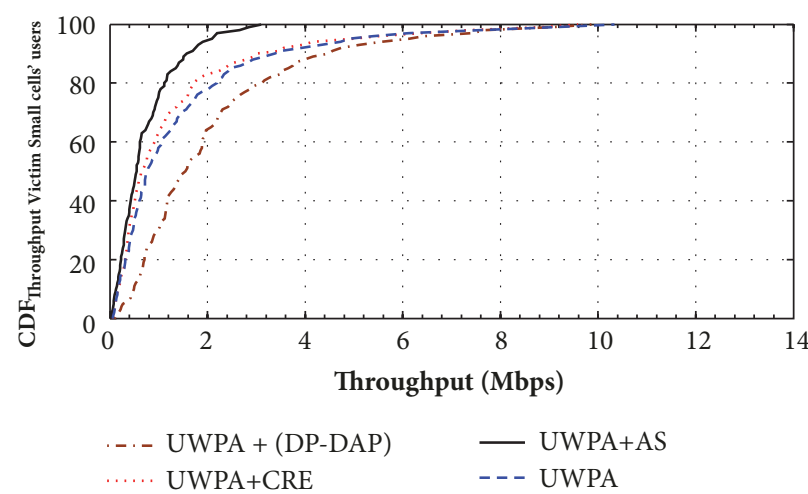

(b) $\alpha=0.3$

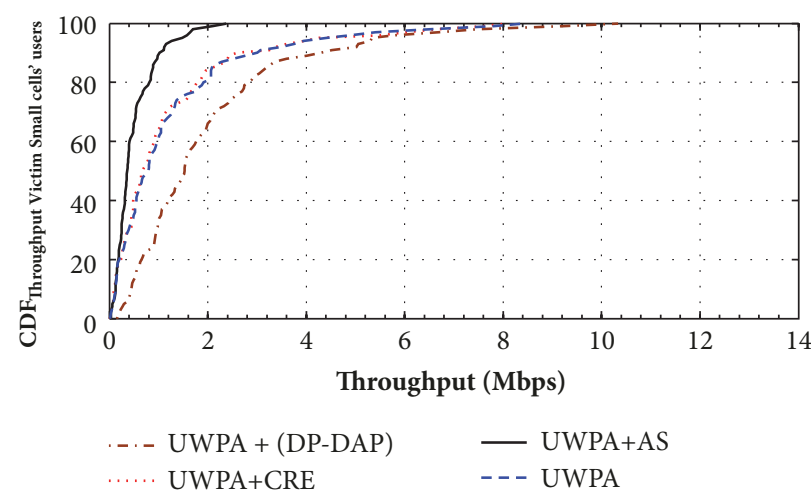

(c) $\alpha=0.2$

FIGURE 12: Average throughput for victim small cells' users (CDF).

To conclude, for bias $=3 \mathrm{~dB}, \alpha=0.3$ shows the highest improvement against $\alpha=0.4$ and 0.2 . However, our proposed $(U W P A+D P-D A P)$ outperforms other schemes regardless of $\alpha$ values. When $\alpha=0.3,50 \%$ of small-cell users have an average throughput equal to or less than $1.909 \mathrm{Mbps}$ compared to $57 \%$ in ((UWPA + CRE), UWPA only) and $78 \%$ for $(U W P A+A S)$.

\section{Conclusions}

This article proposed a novel H-CRAN cooperative interference mitigation method in the time domain. The study firstly reviewed a previously proposed user weighted probabilitybased spectrum sharing algorithm (UWPA) that divides the spectrum into shared and dedicated partitions and allocate them efficiently to each cell in the network. The UWPA works jointly with the proposed DP-DAP algorithm in this article to provide comprehensive solution for intertier interference mitigation. The proposed DP-DAP introduces a novel diverse-ABSF pattern scheme based on the exiting ABSF scheme. The main goal is to enhance the small-cell users SINR and overall system throughput. In the previous contribution made in the spectrum division part (UWPA), the proposed UWPA enhanced the small-cell user's throughput by around $15 \%$ in average compared to the other stateof-the-art schemes. Other enhancements are also observed such as the increase in the macrousers' throughput which is around $10 \%$ compared to other schemes, while the UWPA resulted in $15 \%$ enhancement in macrousers' satisfaction ratio. UWPA is further enhanced by applying (DP-DAP) to improve small-cell user's SINR. In the DP-DAP technique, an intelligent utilization of time slots is performed that can be processed at the RIE along with the exploitation of new technology of directive antennas in order to achieve even higher performance in terms SINR and data rate at the macro- and small-cell users. For example, the joint (DPDAP + UWPA) is observed to result in 56\% improvement in average user data rate and $41 \%$ in number of scheduling times compared to (UWPA+classic ABSF) scheme. In addition, the (DP-DAP + UWPA) can offer up to $7 \%$ and $28 \%$ increase in the small-cell users SINR when compared to the (UWPA + CRE) and (UWPA+AS) schemes, respectively.

\section{Data Availability}

The data used to support the findings of this study are available from the corresponding author upon request.

\section{Conflicts of Interest}

The authors declare that they have no conflicts of interest. 


\section{Authors' Contributions}

Imad Al-Samman is the main author of this paper and Imad proposed the main idea of the article. Reham Almesaeed has helped in developing the proposed algorithm, channel modelling, and writing the paper. All authors read and approved the final manuscript.

\section{Acknowledgments}

This work is funded by Engineering and Physical Sciences Research Council (EPSRC-UK), NEC, Centre for Doctoral Training in communication (CDT), University of Bristol, UK, and the University of Bahrain.

\section{References}

[1] C. Lini, J. Huang, R. Duan, C. Cui, and J. Jiang, Recent Progress on C-RAN Centralization and Cloudification, IEEE Special Section on Recent Advances in Cloud radio Access Network, 2014.

[2] C-RAN: The Road Towards Green RAN. China Mobile Research Institute,

[3] M. Peng, Y. Li, J. Jiang, J. Li, and C. Wang, "Heterogeneous cloud radio access networks: a new perspective for enhancing spectral and energy efficiencies," IEEE Wireless Communications Magazine, vol. 21, no. 6, pp. 126-135, 2014.

[4] M. Gerasimenko, D. Moltchanov, R. Florea et al., "Cooperative radio resource management in heterogeneous cloud radio access networks," IEEE Access, vol. 3, pp. 397-406, 2015.

[5] M. Peng, Y. Li, Z. Zhao, and C. Wang, System Architecture and Key technologies for $5 G$ Heterogeneous Cloud Radio Access Networks, IEEE Network, 2015.

[6] M. Matinmikko, H. Okkonen, M. Palola, S. Yrjölä, P. Ahokangas, and M. Mustonen, "Spectrum sharing using licensed shared access: the concept and its workflow for LTE-Advanced networks," IEEE Wireless Communications Magazine, vol. 21, no. 2, pp. 72-79, 2014.

[7] R. E. Learned, S. E. Johnston, and N. J. Kaminski, "Cognitive coexistence: A throughput study of MUD-enhanced opportunistic spectrum access," in Proceedings of the 2013 47th Asilomar Conference on Signals, Systems and Computers, pp. 1455-1462, USA, November 2013.

[8] X. Costa-Perez, J. Swetina, T. Guo, R. Mahindra, and S. Rangarajan, "Radio access network virtualization for future mobile carrier networks," IEEE Communications Magazine, vol. 51, no. 7, pp. 27-35, 2013.

[9] P. Demestichas, A. Georgakopoulos, D. Karvounas et al., "5G on the Horizon: key challenges for the radio-access network," IEEE Vehicular Technology Magazine, vol. 8, no. 3, pp. 47-53, 2013.

[10] K. Sakaguchi, S. Sampei, H. Shimodaira, R. Rezagah, G. K. Tran, and K. Araki, "Cloud cooperated heterogeneous cellular networks," in Proceedings of the 2013 21st International Symposium on Intelligent Signal Processing and Communication Systems, ISPACS 2013, pp. 787-791, Japan, November 2013.

[11] S. Kim, "Dynamic C-RAN resource sharing scheme based on a hierarchical game approach," EURASIP Journal on Wireless Communications and Networking, vol. 2016, no. 1, pp. 1-12, 2016.

[12] H. Li, D. Liu, Y. Dai, and T. H. Luan, "Engineering searchable encryption of mobile cloud networks: when QoE meets QoP,"
IEEE Wireless Communications Magazine, vol. 22, no. 4, pp. 7480, 2015.

[13] S. Kim, "Learning Based Spectrum Sharing Algorithms by Using Coopetition Game Approach," Wireless Personal Communications, vol. 82, no. 3, pp. 1799-1808, 2015.

[14] J. Pang, J. Wang, D. Wang, G. Shen, Q. Jiang, and J. Liu, "Optimized time-domain resource partitioning for enhanced intercell interference coordination in heterogeneous networks," in Proceedings of the 2012 IEEE Wireless Communications and Networking Conference, WCNC 2012, pp. 1613-1617, France, April 2012.

[15] S. Lembo, P. Lunden, O. Tirkkonen, and K. Valkealahti, "Optimal muting ratio for Enhanced Inter-Cell Interference Coordination (eICIC) in HetNets," in Proceedings of the 2013 IEEE International Conference on Communications Workshops, ICC 2013, pp. 1145-1149, Hungary, June 2013.

[16] H. Zeng, Y. Shi, Y. T. Hou, W. Lou, S. Kompella, and S. F. Midkiff, "An analytical model for interference alignment in Multi-Hop MIMO networks," IEEE Transactions on Mobile Computing, vol. 15, no. 1, pp. 17-31, 2016.

[17] 3GPP Study on small cell enhancement for e-utra and e-utran physical-layer aspects, Study on small cell enhancement for eutra and e-utran physical-layer aspects, 3GPP, 2013.

[18] T. Novlan, J. Andrews, I. Sohn, and R. Ganti, Comparison of fractional frequency reuse approaches in the OFDMA cellular downlink. IEEE Globcom Telecommunication,.

[19] D. Perez, A. Juttner, H. Rivano, and J. Zhang, Dynamic frequency planning versus frequency schemes in OFDMA networks. IEEE Vehicular Technology,

[20] G. Guvenc, M.-R. Jeong, F. Watanabe, and H. Inamura, "A hybrid frequency assignment for femtocells and coverage area analysis for co-channel operation," IEEE Communications Letters, vol. 12, no. 12, pp. 880-882, 2008.

[21] Y. Bai, J. Zhou, and L. Chen, "Hybrid Spectrum Usage for overlaying lTE macrocell and femtocell," IEEE Globcom, 2009.

[22] I. Al-Samman, R. Almesaeed, A. Doufexi, M. Beach, and A. Nix, "User weighted probability algorithm for heterogeneous CRAN interference mitigation," in Proceedings of the 2017 IEEE International Conference on Communications, ICC 2017, France, May 2017.

[23] Z. Wang, H. Li, H. Wang, and S. Ci, "Probability weighted based spectral resources allocation algorithm in Hetnet under CloudRAN architecture," in Proceedings of the 2013 IEEE/CIC International Conference on Communications in China - Workshops, CIC/ICCC 2013, pp. 88-92, China, August 2013.

[24] J. Wang, D. Wang, J. Pang, and G. Shen, "Optimized fairness cell selection for 3gpp lte-a macro-pico HetNets," in IEEE 74th VTC-Fall, United States, San Francisco, 2011.

[25] T. Bu, L. Li, and R. Ramjee, "Generalized proportional fair scheduling in third generation wireless data networks," in Proceedings of the 25th IEEE International Conference on Computer Communications (INFOCOM '06), pp. 1-12, April 2006.

[26] S. Sesia, I. Toufik, and M. Baker, "LTE - The UMTS Long Term Evolution: From Theory to Practice," LTE - The UMTS Long Term Evolution: From Theory to Practice, pp. 1-611, 2009.

[27] System performance of heterogeneous networks with range expansion. 3GPP R1-100142, 2010.

[28] R. Almesaeed, A. S. Ameen, E. Mellios, A. Doufexi, and A. R. Nix, "A proposed 3D extension to the 3GPP/ITU channel model for $800 \mathrm{MHz}$ and $2.6 \mathrm{GHz}$ bands," in Proceedings of the 8th European Conference on Antennas and Propagation, EuCAP 2014, pp. 3039-3043, Netherlands, April 2014. 
[29] ITU-R, Guidelines for evaluation of radio interface technologies for IMT-Advanced, 2009.

[30] C.-N. Lee, J.-H. Lin, C.-F. Wu, M.-F. Lee, and F.-M. Yeh, "A dynamic CRE and ABS scheme for enhancing network capacity in LTE-advanced heterogeneous networks," Wireless Networks, pp. 1-16, 2018.

[31] Evolved Universal Terrestrial Radio Access (EUTRA) and Evolved Universal Terrestrial Radio Access Network (EUTRAN): Overall Description, Stage2, V10.7.0, March 2012. 


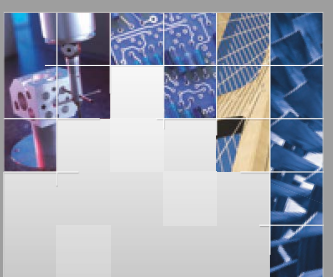

\section{Enfincering}
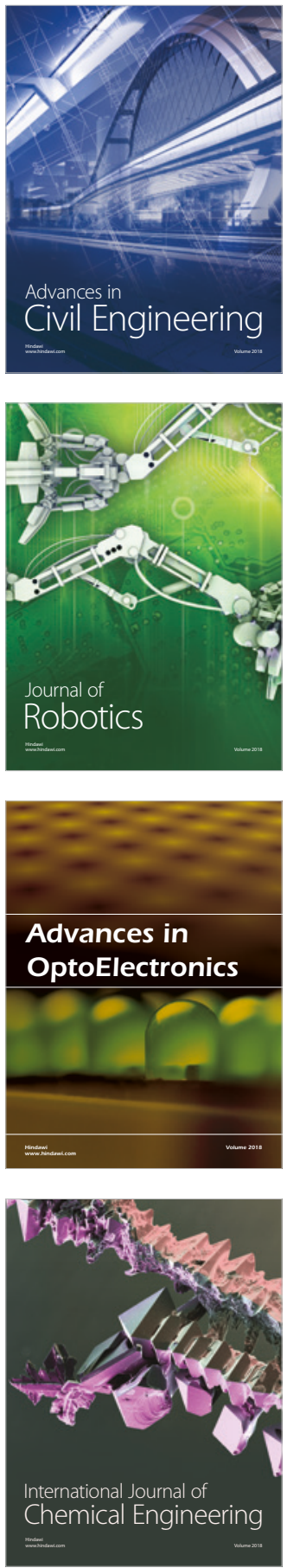

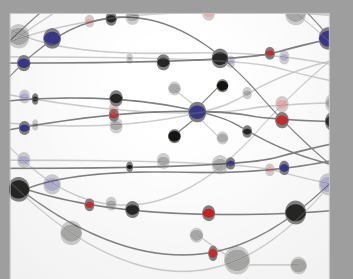

\section{Rotating \\ Machinery}

The Scientific World Journal

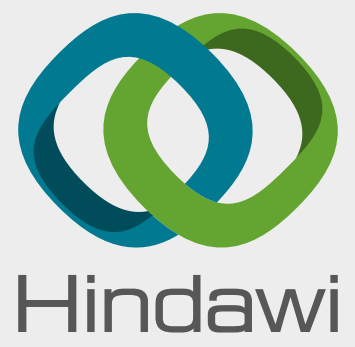

Submit your manuscripts at

www.hindawi.com
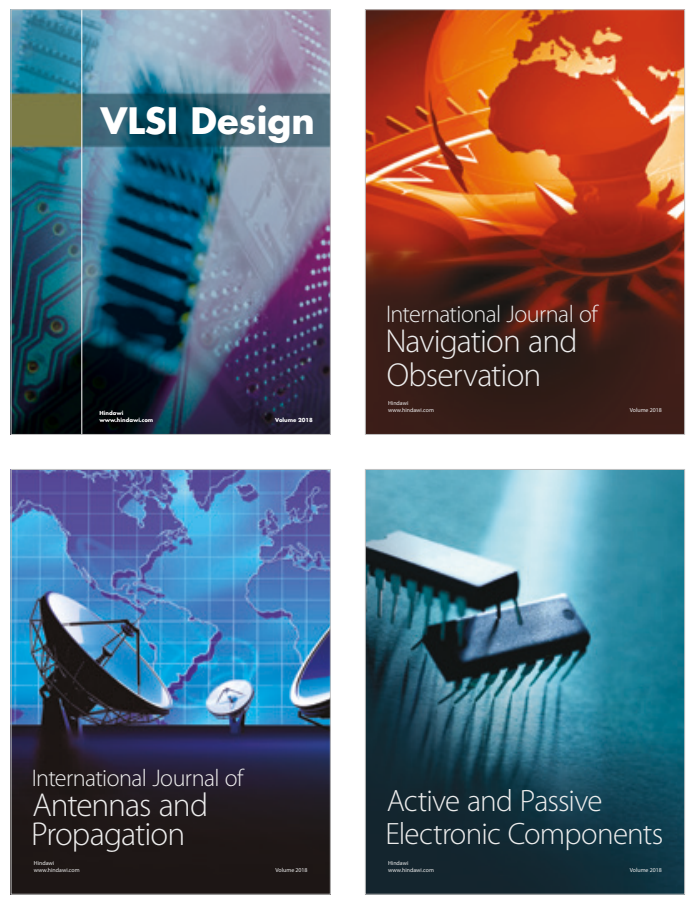
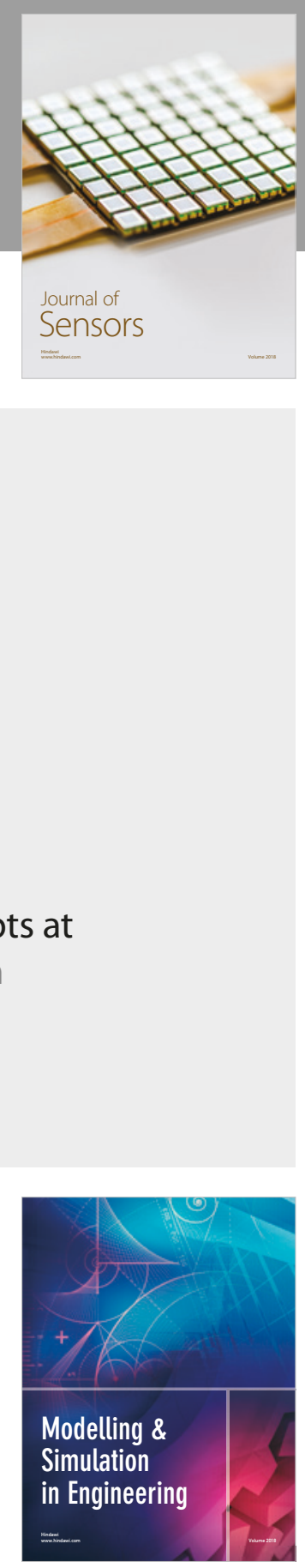

\section{Advances \\ Multimedia}
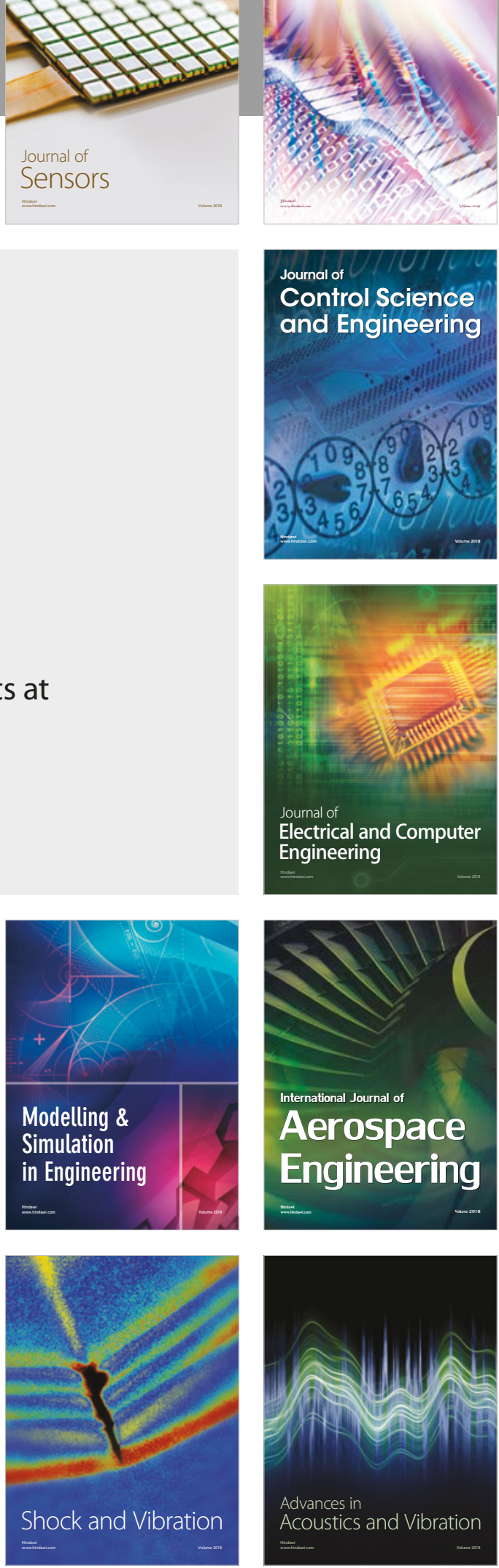